\title{
A New Resultant Vibration Acceleration Model of a Planetary Gear Train and Fault Response Analysis
}

\author{
Rui Meng $\mathbb{D}^{1,2,3}$ Xin Cai $\mathbb{D}^{1,4,5}$ and Xingwen Guo $\mathbb{i D}^{1,4,5}$ \\ ${ }^{1}$ College of Mechanics and Materials, Hohai University, Nanjing 210098, China \\ ${ }^{2}$ AHUT Engineering Research Institute, Anhui University of Technology, Maanshan 243002, China \\ ${ }^{3}$ School of Mechanical Engineering, Anhui University of Technology, Maanshan 243002, China \\ ${ }^{4}$ Jiangsu Province Wind Power Structural Engineering Research Center, Hohai University, Nanjing 210098, China \\ ${ }^{5}$ Collaborative Innovation Centre for Developing and Protecting Coastal Area, Hohai University, Nanjing 210098, China \\ Correspondence should be addressed to Xin Cai; xcai@hhu.edu.cn
}

Received 12 August 2021; Revised 13 December 2021; Accepted 21 December 2021; Published 27 February 2022

Academic Editor: F. Viadero

Copyright (c) 2022 Rui Meng et al. This is an open access article distributed under the Creative Commons Attribution License, which permits unrestricted use, distribution, and reproduction in any medium, provided the original work is properly cited.

\begin{abstract}
A new resultant vibration acceleration model is proposed to reveal its signal characteristics more accurately in the healthy and faulty state. First, an analytical lateral-torsional coupled dynamic model is developed with consideration of time-varying mesh stiffness and damping, static transmission error, and gear backlash. Then, the effect of gear backlash and damping on the system is analyzed, and a numerical velocity signal in the healthy and faulty state is carried out. Considering the effects of transmission paths, the resultant acceleration signal along the vertical direction is constructed as the weighted vibration summation. This signal includes a vertical component of the vibration along the meshing lines of both sun-planet and ring-planet pairs. Moreover, it also contains the vertical component of the planet gears, sun gear, and planet carrier acceleration relative to their own supporting bearings. Finally, the simulation results from the resultant signal model are experimentally validated and analyzed in both time and frequency domains.
\end{abstract}

\section{Introduction}

Planetary gear train has the compact structure and desirable transmission efficiency. Therefore, it is widely used in mechanical transmission systems. Compared with fixed-shaft gear train, planetary gears simultaneously mesh with the sun gear and the ring gear, which leads to the relatively complex load sharing behavior [1-4] and spectral structure of vibration [5-8]. For load sharing behavior, researchers have carried out meaningful basic research on it. For instance, the influence of carrier and gear manufacturing errors on the static load sharing was studied [1]. Singh successively carried out systematic research on load sharing behavior and developed the load sharing map $[2,3]$. Influence of errors on load sharing under different mesh phasing was analyzed [4]. The rotation of the planet carrier also modulates the received signal by the fixed sensor. Therefore, the vibration response of a planetary gear train is more complex than that of the fixed-shaft gear train. Correspondingly, fault diagnosis for a planetary gear train is more difficult than the one of the fixed-shaft gear train. Because the dynamics model in both healthy and fault conditions can be established based on the fundamental analysis of the gear mesh mechanism, fault symptoms can be revealed and further summarized for fault detection and diagnosis. In dynamics model of a planetary gear train, lumped parameter modeling (LPM) [9-14] was widely applied, and its basic application was related to the spectral analysis, such as sideband activity in frequency domain [5-7] and envelope spectrum analysis [8]. Vibration analysis based on LPM typically involves the following four aspects, including model simplifications, establishment of the gear failure model, evaluation of gear mesh stiffness, and construction of the resultant signal. Strictly speaking, each component (sun gear, planetary gear, ring gear, and planet carrier) has three degrees of freedom: a single rotational angular displacement and two lateral translations in the $x$ - 
and $y$-directions. However, some researchers have simplified the degree of freedom of the model to reduce the complexity of the differential equation. A torsional vibration model for planetary gear fault detection was established [15]. Cheng et al. [16] proposed a model that considered the rotational angular displacement of each component. Lei et al. [17] built a model that included three degrees of freedom of the sun gear without considering lateral translations of other components in the $x$ - and $y$-directions. In the spring-damper system, there are multiple nonlinear parameters, such as the time-varying mesh stiffness and damping, static transmission error, and gear backlash. However, some nonlinear parameters are not taken into account in order to solve the dynamic equations more quickly. For example, static transmission error and gear backlash [17-20] and damping and gear backlash [21] were neglected. The widely studied gear defect models include shape deviations and mounting errors [22], gear tooth crack [18, 21, 23-26], gear tooth pitting or spalling $[23,27-30]$, tooth wear $[8,31]$, and tooth tip chipping $[32,33]$. Calculation results of mesh stiffness are a prerequisite for solving dynamic equations $[34,35]$. One significant difference between the healthy and the faulty gear can be found in their mesh stiffness [18, 36, 37]. Currently, four methods are mainly used to evaluate the mesh stiffness: the square wave method, potential energy method, finite element method, and experimental method. For instance, the square wave method was applied to evaluate the mesh stiffness of healthy planetary gears [38], tooth cracking $[39,40]$, tooth pitting [41], manufacturing error [20], and tooth profile error [42]. The potential energy method was used to evaluate the mesh stiffness of healthy planetary gears [19], tooth cracking [18, 24], and tooth pitting [29]. The finite element method depends on the finite element software to calculate the mesh stiffness, where the sun-planet mesh stiffness and the ring-planet mesh stiffness are uncoupled. The distinctive feature of the finite element method lies in evaluating the gear mesh stiffness for any gear profile type. For example, mesh stiffness of healthy and cracked gear [21] was evaluated by finite element method. Park et al. [43] proposed experimental procedures to estimate the gear mesh stiffness of rotating gears under faulty states.

The vibration value of each component in the planetary gear train can be obtained by solving its differential dynamic equations. The signal perceived by the fixed sensor is a resultant signal containing the attenuated vibrations of multiple components. Moreover, planet carrier rotation may cause the timevarying distances between vibration sources and a transducer. Therefore, it is necessary to construct the resultant signal while accounting for the transmission path effect. Many researchers have made useful contributions in this field. He et al. [44] established the resultant vibration signal, where the proportional coefficients were used to establish the relation between the vibration responses and gear mesh forces. Moreover, three transmission paths were described by the Fourier series whose fundamental frequency was the rotating frequency of the planet carrier. Based on model [44], dynamics modeling with a floating sun gear was established, and vibration modulation signal was further studied $[45,46]$. The resultant vibration signal [20] was modeled as the weighted summation of the dynamic mesh forces of all planet gears, where constant values were facilitated to establish the relation between the gear mesh forces and the resultant acceleration. Moreover, a modified Hanning function was applied to represent the effect of the transmission path. The resultant vibration signal was also modeled as the weighted summation of the vibration acceleration of all planet gears [18], where a modified Hamming function was used to represent the effect of the transmission path. The dynamic behavior of planetary gearbox based on improved Hanning function was simulated [47], where resultant vibration signal only contained the vibrations of the four planetary gears. Liu et al. [19] constructed the transducer-perceived vibration signal containing vibration information of the sun gear, planet gears, and the ring gear. The authors also analyzed the different transmission paths on the resultant vibration signals. Liu et al. [21] pointed out that resultant vibration contained the acceleration vibration signals of internal and external mesh pairs. Moreover, the authors employed the improved Hamming function to reflect the influence of the transfer path. Lei et al. [17] proposed that the vibration signal model included vibration signals along the meshing lines as well as sun gear's vibration signal, where the amplitude modulation function based on rotating frequency of the planet carrier was used to reflect the transmission path effect. Different from expressing transfer path with window functions above, Zghal B et al. [48] implicitly reflected the variable transmission path by expressing the displacement of each component at the transducer location before the resolution of the equation of motion, where the resultant vibration signal was obtained at the transducer location as the summation of all vibration signals. Moreover, the vibration characteristics in healthy condition were validated by experimental laboratory data in frequency domain.

Based on the conducted literature review, it can be found that the previous studies on the resultant vibration of planetary gear train can be roughly clarified into two groups: one group focalized the vibration of the internal and external meshing lines, but not comprehensively took the vibration of each component relative to its own bearing into account. The other group was in consideration of the vibration of some components relative to its own bearing, but it did not consider the vibration of the internal and external meshing lines. In view of this, the present study aims to construct a more comprehensive resultant vibration model of a planetary gear train based on its dynamic equations, where dynamic equations contain multiple kinds of nonlinear parameters (time-varying mesh stiffness and damping, static transmission error, and gear backlash). The resultant vibration model mainly contains the vibration signals along the meshing lines of both the sun-planet and the ringplanet pairs, and vibration information of the planet gears, sun gear, and planet carrier relative to their supporting bearings, where the modified Hamming function and attenuation coefficients are used to reflect the effect of transmission paths on transducer-perceived signal.

The structure of this article is as follows. Lateral-torsional coupled dynamic model of a planetary gear train, mesh stiffness evaluation is shown in Section 2. Then, effect analysis of gear backlash and damping, as well as the numerical simulation of velocity signals in healthy and faulty conditions, is carried out. A new resultant vibration model of a planetary gear train is 
proposed in Section 3. To validate the simulation results in both time and frequency domains, an experimental test is carried out in Section 4. Finally, conclusions are drawn in Section 5.

\section{Dynamic Simulation}

2.1. Dynamics Model of a Planetary Gear Train. Lumped parameter dynamic model is widely used to analyze the vibration characteristics of planetary gear train. Herein, a lateral-torsional coupled dynamic model of a planetary gear train is presented to simulate vibration signals of planetary gear train, which is shown in Figure 1. The model consists of a single sun gear $(s)$, ring gear $(r)$, planet carrier $(c)$, and $N$ planet gears $(p n(n=1,2, \cdots, N))$. Oxy is defined as the rotating coordinate system revolving at the speed of the carrier $\omega_{c}$ with $x$-axis passing through the center of the $1^{\text {st }}$ planet. $O X Y$ is the fixed coordinate system, where the vibration sensor is located.

Each component $i(i=s, r, c, p n)$ has three degrees of freedom, i.e., two translation motions $\left(x_{i}, y_{i}\right)$ and one torsion motion $\left(u_{i}\right)$. The translational bearing stiffness is represented by $k_{s}, k_{r}, k_{o} k_{p}$ and the torsional bearing stiffness is denoted by $k_{s u}, k_{r u}, k_{c u}, k_{p u}$. The translational bearing damping is represented by $c_{s}, c_{r}, c_{c}, c_{p}$ and the torsional bearing damping is denoted by $c_{s u}, c_{r u}, c_{c u}, c_{p u}$. The internal and external meshing pairs are represented by the virtual spring-damper system with time-varying mesh stiffness $\left(k_{r n}(t), k_{s n}(t)\right)$ and damping $\left(c_{r n}(t), c_{s n}(t)\right)$, static transmission error $\left(e_{r n}(t)\right.$, $\left.e_{s n}(t)\right)$, and gear backlash $\left(b_{r n}, b_{s n}\right)$ along the line of action.

By applying the $2^{\text {nd }}$ Newtonian law, the differential motion equations of a planetary gear train can be derived. Equations of motion for the planet carrier are written as follows:

$$
\left\{\begin{array}{l}
m_{c}\left(\ddot{x}_{c}-2 \omega_{c} \dot{y}_{c}-\omega_{c}^{2} x_{c}\right)+\sum_{n=1}^{N}\left[k_{p} \delta_{c n x}+c_{p} \dot{\delta}_{c n x}\right]+k_{c} x_{c}+c_{c} \dot{x}_{c}=0 \\
m_{c}\left(\ddot{y}_{c}+2 \omega_{c} \dot{x}_{c}-\omega_{c}^{2} y_{c}\right)+\sum_{n=1}^{N}\left[k_{p} \delta_{c n y}+c_{p} \dot{\delta}_{c n y}\right]+k_{c} y_{c}+c_{c} \dot{y}_{c}=0 \\
\left(\frac{I_{c}}{r_{c}^{2}}\right) \ddot{u}_{c}+\sum_{n=1}^{N}\left[k_{p} \delta_{c n u}+c_{p} \dot{\delta}_{c n u}\right]+k_{c u} u_{c}+c_{c u} \dot{u}_{c}=-\frac{T_{c}}{r_{c}} .
\end{array}\right.
$$

Equations of motion for the ring gear are written as follows:

$$
\left\{\begin{array}{c}
m_{r}\left(\ddot{x}_{r}-2 \omega_{c} \dot{y}_{r}-\omega_{c}^{2} x_{r}\right)-\sum_{n=1}^{N}\left[k_{r n}(t) \delta_{r n}+c_{r n}(t) \dot{\delta}_{r n}\right] \sin \beta_{r n}+k_{r} x_{r}+c_{r} \dot{x}_{r}=0 \\
m_{r}\left(\ddot{y}_{r}+2 \omega_{c} \dot{x}_{r}-\omega_{c}^{2} y_{r}\right)+\sum_{n=1}^{N}\left[k_{r n}(t) \delta_{r n}+c_{r n}(t) \dot{\delta}_{r n}\right] \cos \beta_{r n}+k_{r} y_{r}+c_{r} \dot{y}_{r}=0 \\
\left(\frac{I_{r}}{r_{r}^{2}}\right) \ddot{u}_{r}+\sum_{n=1}^{N}\left[k_{r n}(t) \delta_{r n}+c_{r n}(t) \dot{\delta}_{r n}\right]+k_{r u} u_{r}+c_{r u} \dot{u}_{r}=0 .
\end{array}\right.
$$

Equations of motion for the sun gear are given as follows:

$$
\left\{\begin{array}{c}
m_{s}\left(\ddot{x}_{s}-2 \omega_{c} \dot{y}_{s}-\omega_{c}^{2} x_{s}\right)-\sum_{n=1}^{N}\left[k_{s n}(t) \delta_{s n}+c_{s n}(t) \dot{\delta}_{s n}\right] \sin \beta_{s n}+k_{s} x_{s}+c_{s} \dot{x}_{s}=0 \\
m_{s}\left(\ddot{y}_{s}+2 \omega_{c} \dot{x}_{s}-\omega_{c}^{2} y_{s}\right)+\sum_{n=1}^{N}\left[k_{s n}(t) \delta_{s n}+c_{s n}(t) \dot{\delta}_{s n}\right] \cos \beta_{s n}+k_{s} y_{s}+c_{s} \dot{y}_{s}=0 \\
\left(\frac{I_{s}}{r_{s}^{2}}\right) \ddot{u}_{s}+\sum_{n=1}^{N}\left[k_{s n}(t) \delta_{s n}+c_{s n}(t) \dot{\delta}_{s n}\right]+k_{s u} u_{s}+c_{s u} \dot{u}_{s}=\frac{T_{s}}{r_{s}} .
\end{array}\right.
$$




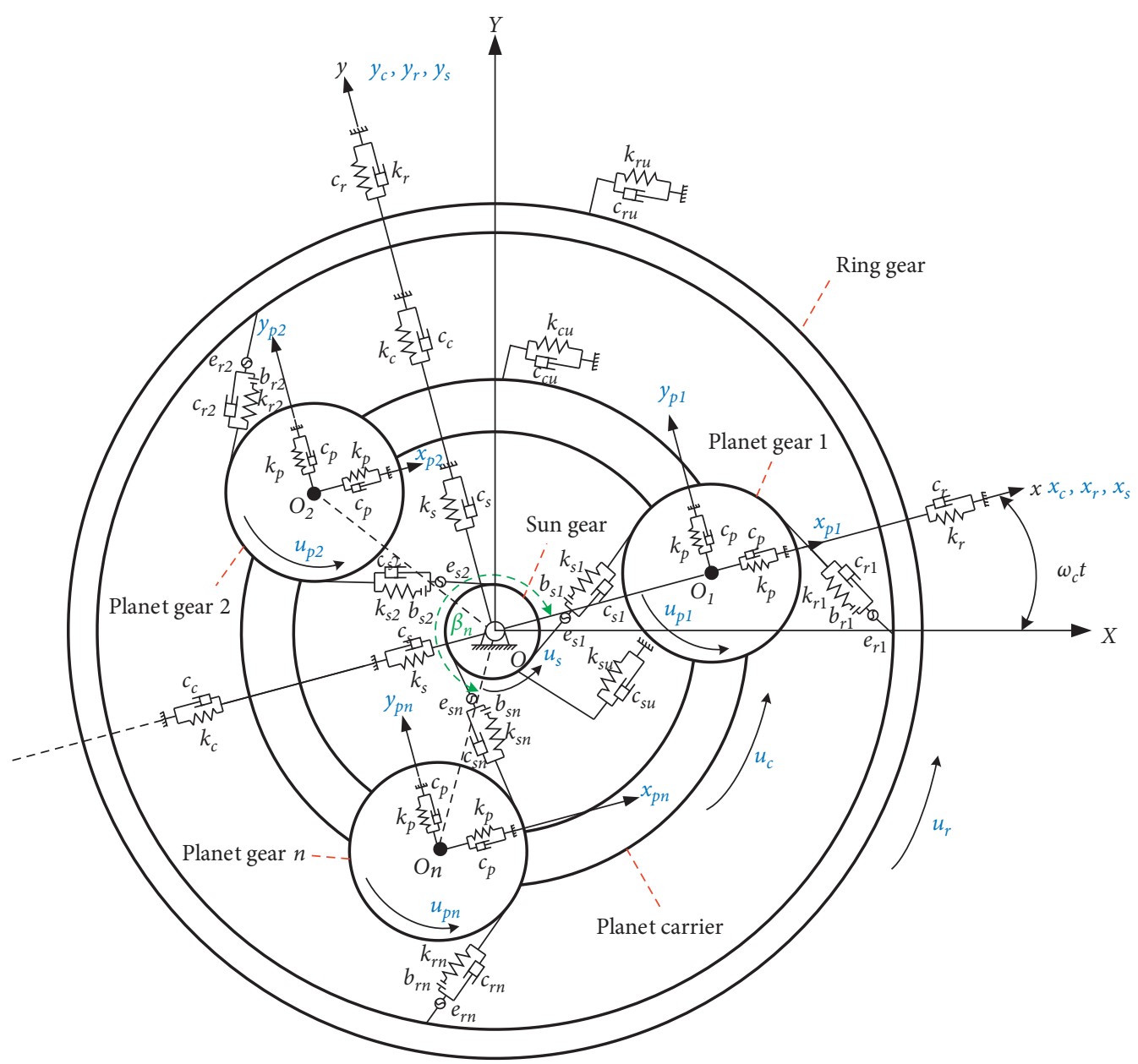

FIgURE 1: Lateral-torsional coupled dynamic model of a planetary gear train.

Equations of motion for the $n^{\text {th }}$ planet are expressed as follows:

$$
\left\{\begin{array}{l}
m_{p}\left(\ddot{x}_{p n}-2 \omega_{c} \dot{y}_{p n}-\omega_{c}^{2} x_{p n}\right)+\left[k_{s n}(t) \delta_{s n}+c_{s n}(t) \dot{\delta}_{s n}\right] \sin \beta_{s n}+\left[k_{r n}(t) \delta_{r n}+c_{r n}(t) \dot{\delta}_{r n}\right] \sin \beta_{r n}-k_{p} \delta_{c n x}-c_{p} \dot{\delta}_{c n x}=0 \\
m_{p}\left(\ddot{y}_{p n}+2 \omega_{c} \dot{x}_{p n}-\omega_{c}^{2} y_{p n}\right)-\left[k_{s n}(t) \delta_{s n}+c_{s n}(t) \dot{\delta}_{s n}\right] \cos \beta_{s n}-\left[k_{r n}(t) \delta_{r n}+c_{r n}(t) \dot{\delta}_{r n}\right] \cos \beta_{r n}-k_{p} \delta_{c n y}-c_{p} \dot{\delta}_{c n y}=0 \\
\left(I_{p} / r_{p}^{2}\right) \ddot{u}_{p n}+\left[k_{s n}(t) \delta_{s n}+c_{s n}(t) \dot{\delta}_{s n}\right]-\left[k_{r n}(t) \delta_{r n}+c_{r n}(t) \dot{\delta}_{r n}\right]=0
\end{array}\right.
$$

where $I_{s}, I_{c}, I_{r}$, and $I_{p}$ denote the moment of inertia of the sun gear, the planet carrier, the ring gear, and the planet gear, respectively. Similarly, the mass of each component is defined as $m_{s}, m_{c}, m_{r}$, and $m_{p}$, respectively. Parameters $r_{s}, r_{r}$, and $r_{p}$ represent the base circle radii of the sun gear, the ring gear, and the planet gear, while $r_{c}$ represents the radius of the pin circle at the carrier. Parameters $T_{s}$ and $T_{c}$ denote the external torques on the sun gear and planet carrier, respectively. $\beta_{s n}=\beta_{n}-\alpha_{s} ; \beta_{r n}=\beta_{n}+\alpha_{r}$, where $\alpha_{s}$ and $\alpha_{r}$ are the meshing angles of sun-planet and ring-planet pairs. $\beta_{n}=2 \pi(n-1) N^{-1}$, where $N$ is the total number of planets. Parameters $\delta_{c n x}, \delta_{c n y}$, and $\delta_{c n u}$ are relative displacements along the $x, y$ and $u$ directions between the planet and planet carrier. Parameters $\delta_{s n}$ and $\delta_{r n}$ denote relative displacements along the meshing lines of the sun-planet and the ringplanet, respectively.

$$
\left\{\begin{array}{l}
\delta_{c n x}=x_{c}-x_{p n}-u_{c} \sin \beta_{n}, \\
\delta_{c n y}=y_{c}-y_{p n}+u_{c} \cos \beta_{n} \\
\delta_{c n u}=\left(x_{p n}-x_{c}\right) \sin \beta_{n}+\left(y_{c}-y_{p n}\right) \cos \beta_{n}+u_{c} \\
\delta_{s n}=\left(x_{p n}-x_{s}\right) \sin \beta_{s n}+\left(y_{s}-y_{p n}\right) \cos \beta_{s n}+u_{s}+u_{p n}-e_{s n} \\
\delta_{r n}=\left(x_{p n}-x_{r}\right) \sin \beta_{r n}+\left(y_{r}-y_{p n}\right) \cos \beta_{r n}+u_{r}-u_{p n}-e_{r n} .
\end{array}\right.
$$


Equations (1)-(4) can be combined and rewritten in the matrix form:

$M \ddot{\phi}+\left(C_{B}+C_{M}+\omega_{c} W\right) \dot{\phi}+\left(K_{B}+K_{M}-\omega_{c}^{2} K_{C}\right) \phi=F_{E}+F_{I}$,

where $\phi$ is the general coordinates vector, $M$ is the mass matrix, $C_{B}$ and $C_{M}$ are the bearings damping matrix and the mesh damping matrix, $W$ is the gyroscopic matrix, $K_{B}$ and $K_{M}$ are the bearings stiffness matrix and the mesh stiffness matrix, $K_{C}$ is the centripetal stiffness matrix, $F_{E}$ is the external force vector, and $F_{I}$ is the internal excitation force vector induced by the transmission errors. In addition, according to Rayleigh damping formula, $C_{B}+C_{M}=\gamma_{1} \cdot M+\gamma_{2} \cdot\left(K_{B}+K_{M}\right)$, where $\gamma_{1}=34.616$ and $\gamma_{2}=2.2942^{*} 10^{-5}$ are mass proportionality and stiffness proportionality coefficients, respectively. Herein, the matrices in equation (6) can be found in the Appendix. Further, the gear backlash is also taken into account by using gear backlash function, $f\left(\delta_{L n}, b_{L n}\right)(L=r, s)$, which can be expressed by the following equation [49]:

$$
f\left(\delta_{L n}, b_{L n}\right)= \begin{cases}\delta_{L n}-\frac{b_{L n}}{2}, & \delta_{L n}>\frac{b_{L n}}{2}, \\ 0, & \left|\delta_{L n}\right| \leq \frac{b_{L n}}{2}, \\ \delta_{L n}+\frac{b_{L n}}{2}, & \delta_{L n}<\frac{-b_{L n}}{2},\end{cases}
$$

where $b_{L n}(L=r, s)$ is the gear backlash of the internal and the external meshing gear pairs. In this article, $b_{L n}(L=r, s)$ is set to be $30 \mu \mathrm{m}$ and the backlash function can be introduced into the dynamic model only by replacing $\delta_{r n}$ and $\delta_{s n}$ in equations (2)-(4) with $f\left(\delta_{r n}, b_{r n}\right)$ and $f\left(\delta_{s n}, b_{s n}\right)$, respectively. Dynamics equations above can be solved by a fourth-order variable-step Runge-Kutta method to obtain the vibration signals of the planetary gear train.

\subsection{Mesh Stiffness and Static Transmission Error Evaluation.}

Tooth fracture is one of the common failure modes in a gear transmission system. In the dynamic model described in Section 2.1, the gear mesh interface is modeled as a springdamper system. When a spur gear pair meshes, the tooth contact number and the tooth mesh position change. This leads to a periodic variation in the gear mesh stiffness. When a tooth breaks, the mesh stiffness of the gear decreases. Consequently, the vibration properties of the gear system are changed. To comprehensively understand the vibration properties of a planetary gear set, it is essential to effectively evaluate the mesh stiffness. Herein, the parameters of the planetary gear train are listed in Table 1 . The planetary gear train has four equally spaced planet gears $(N=4)$ with a fixed ring gear. The rotation frequency of the planet carrier is set as $1.08 \mathrm{~Hz}\left(f_{c}=1.08 \mathrm{~Hz}\right)$, and a constant load of $5.71 \mathrm{~N} \mathrm{~m}$ is applied on the planet carrier. Mesh stiffness can be evaluated according to the following equation:

$$
k_{m}=\frac{T_{o r}}{\theta r_{b}^{2}},
$$

where $T_{o r}$ is the input torque, $\theta$ is the relative rotation angle between a gear pair, and $r_{b}$ represents the gear base circle radius. Herein, a three-dimensional finite element model of the sun-planet gear pair with/without a $2.4 \mathrm{~mm}$ broken tooth on the sun gear as well as ring-planet gear pair is established to accurately get the value of $\theta$ and then to obtain the mesh stiffness.

Herein, the external meshing gear pair is taken as an example to illustrate the computation steps of using finite element method (FEM) to obtain mesh stiffness:

(1) Create gear assembly model and define material properties in ABAQUS/Standard. The material of gear is linearly elastic with Young's modulus $E=2.06 \times 10^{11} \mathrm{~Pa}$ and Poisson's ratio $v=0.3$.

(2) Obtain the finite element mesh. The element C3D8R is adopted to mesh the bodies of the engaged gears, which can avoid locking phenomena in the finite element analysis to guarantee the accuracy of the solution [21]. Firstly, gear meshing pairs are roughly meshed as a whole. Next, the elements on the engaging surface mesh more finely to obtain well element distribution, which can guarantee the calculation precision of stress and angle $\theta$ more accurately. Finite element mesh of healthy sunplanet is shown in Figure 2(a), where the total elements number is 117726 , and the total nodes number is 157259 .

(3) Set the minimum time interval and interaction surfaces. $1 / 40$ of mesh period is set as the minimum time interval in the analysis step, and the sun gear and the planet gears are selected as the active and driven surfaces, respectively, to establish the interaction surfaces, which are shown in Figure 2(b).

(4) Obtain mesh stiffness. Adjust the gear pairs, so that they are just engaged. Next, the planetary gear is fixed, and the torque is applied to the sun gear. The meshing gear pair will produce elastic deformation and stress, which is shown in Figure 2(c). The value of $\theta$ can be obtained, and the value of mesh stiffness at the first mesh point can be further calculated by equation (8). In the next time interval, planetary gear is fixed after rotating at a small angle. It is notable that the sun gear always keeps meshing with the planetary gear due to the interaction surfaces applied in Step 3. In a similar way, the mesh stiffness at the second mesh point can be obtained. By analogy, all mesh stiffness values are obtained.

Finite element mesh of broken sun-planet (as well as the ring-planet) is shown in Figures 2(d) and 2(e), and interaction surfaces of ring-planet are shown in Figure 2(f). The external and internal mesh stiffness is shown in Figure 3(a), where the planetary gears and ring are healthy, so $k_{r 1}=k_{r 2}=k_{r 3}=k_{r 4}$. The broken tooth of the sun gear makes the angle $\theta$ increase under the action of the same torque, so that the stiffness value 
TABle 1: Parameters of the planetary gear train.

\begin{tabular}{|c|c|c|c|c|}
\hline & Sun gear & Ring gear & Planet carrier & Planet gear \\
\hline Module (mm) & 1 & 1 & - & 1 \\
\hline Tooth number & 28 & 100 & - & 36 \\
\hline Tooth width (mm) & 10 & 10 & - & 10 \\
\hline Pressure angle (rad) & 0.349 & 0.349 & - & 0.349 \\
\hline Inertia $\left(\mathrm{kg} \cdot \mathrm{m}^{2}\right)$ & $6.415 \times 10^{-6}$ & $1.417 \times 10^{-3}$ & $3.648 \times 10^{-4}$ & $1.179 \times 10^{-5}$ \\
\hline Mass $(\mathrm{kg})$ & 0.069 & 0.422 & 0.609 & 0.057 \\
\hline Poisson's ratio & 0.3 & 0.3 & 0.3 & 0.3 \\
\hline Young's modulus $(\mathrm{Pa})$ & $2.06 \times 10^{11}$ & $2.06 \times 10^{11}$ & $2.06 \times 10^{11}$ & $2.06 \times 10^{11}$ \\
\hline Torsional bearing stiffness $\left(\mathrm{N} \cdot \mathrm{m}^{-1}\right)$ & 0 & $2 \times 10^{9}$ & 0 & 0 \\
\hline Translational bearing stiffness $\left(\mathrm{N} \cdot \mathrm{m}^{-1}\right)$ & $4.2 \times 10^{7}$ & $3.0 \times 10^{8}$ & $1.8 \times 10^{8}$ & $1.5 \times 10^{7}$ \\
\hline
\end{tabular}

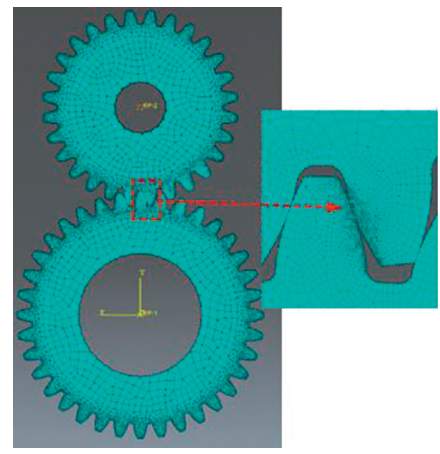

(a)

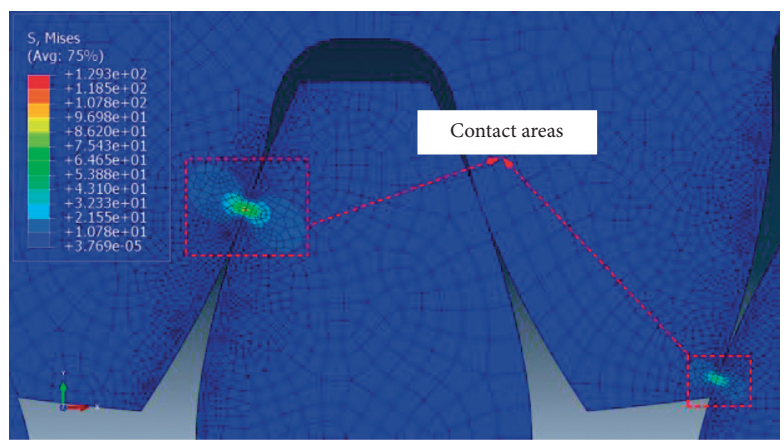

(c)

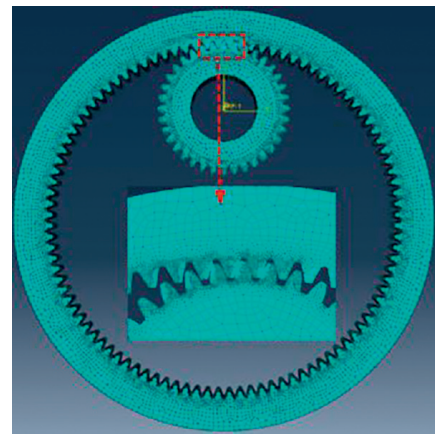

(e)

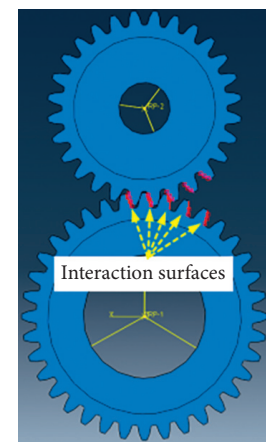

(b)

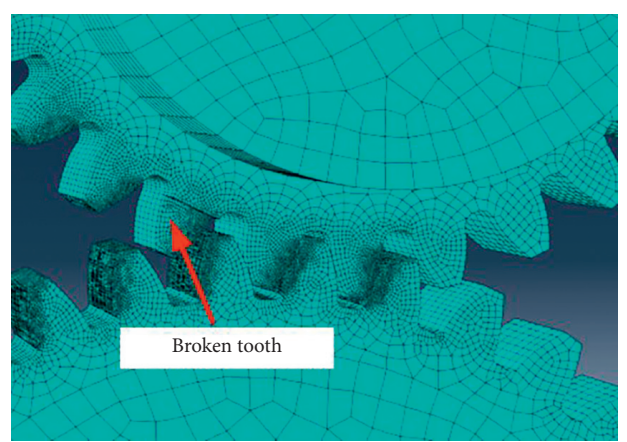

(d)

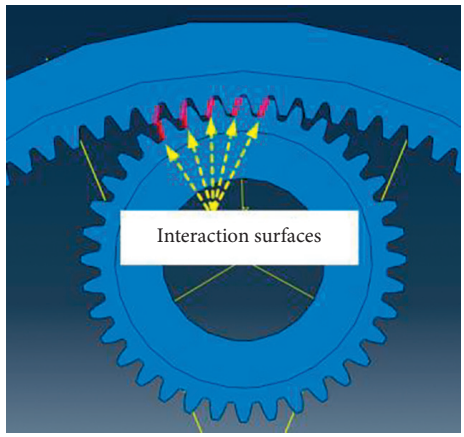

(f)

FIGURE 2: Finite element models for mesh stiffness evaluation. (a) Finite element mesh of healthy sun-planet. (b) Interaction surfaces of healthy sun-planet. (c) Stress diagram of healthy sun-planet. (d) Finite element mesh of broken sun-planet. (e) Finite element mesh of ringplanet. (f) Interaction surfaces of ring-planet. 


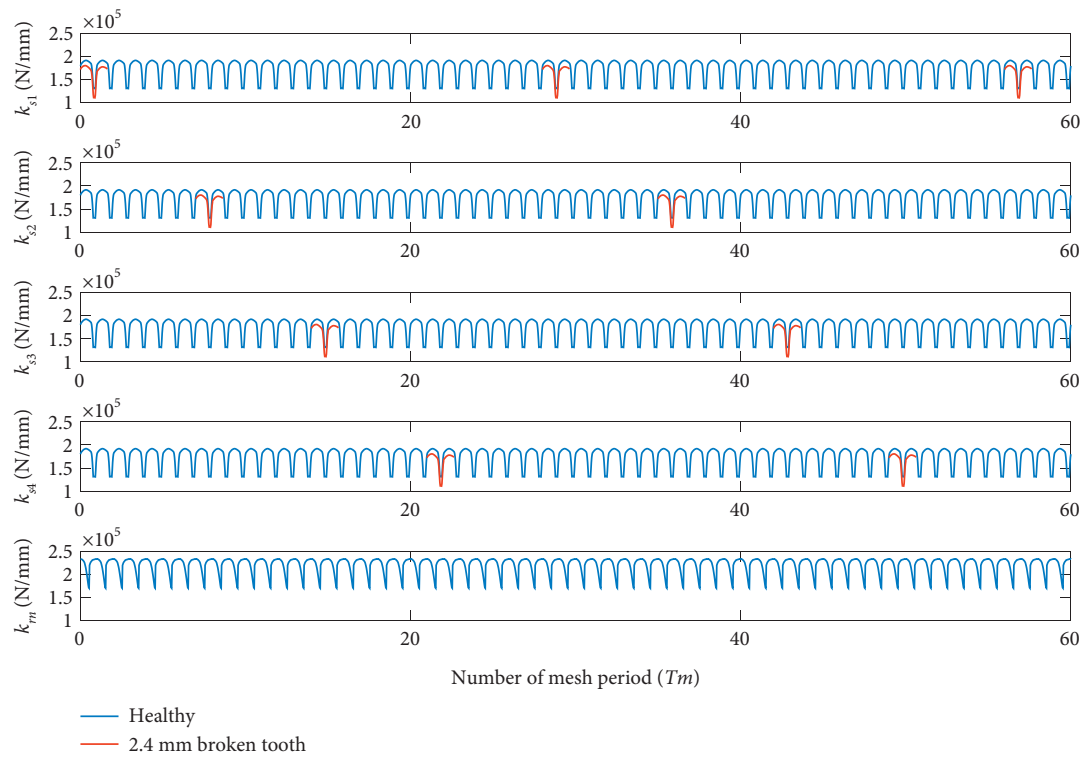

(a)

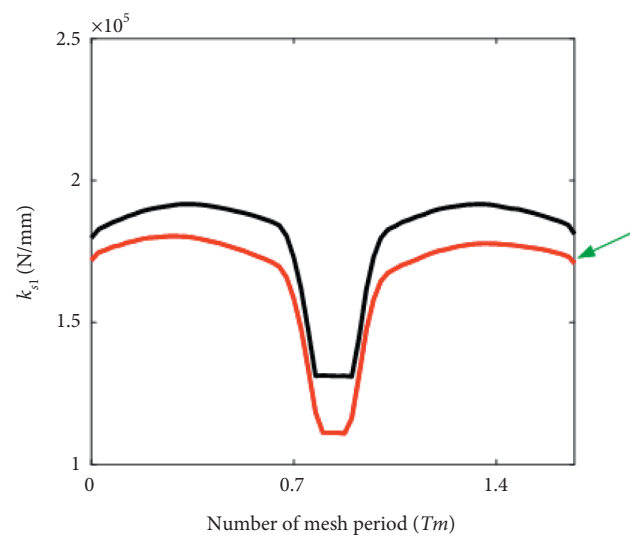

- $2.4 \mathrm{~mm}$ broken tooth

- Healthy

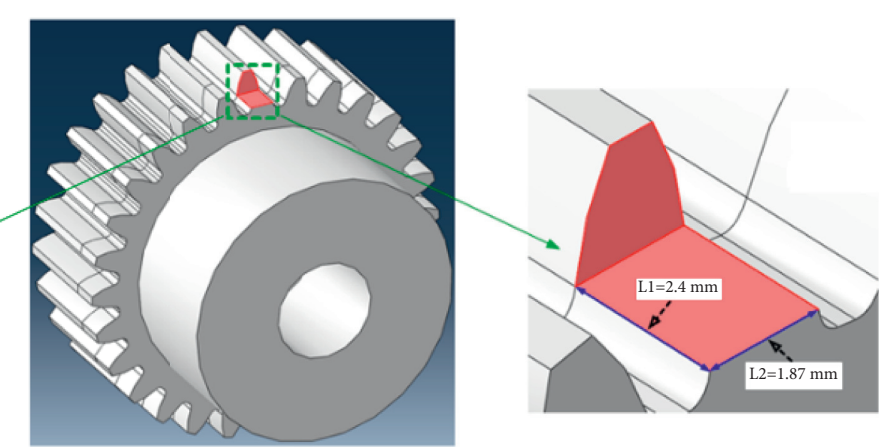

(b)
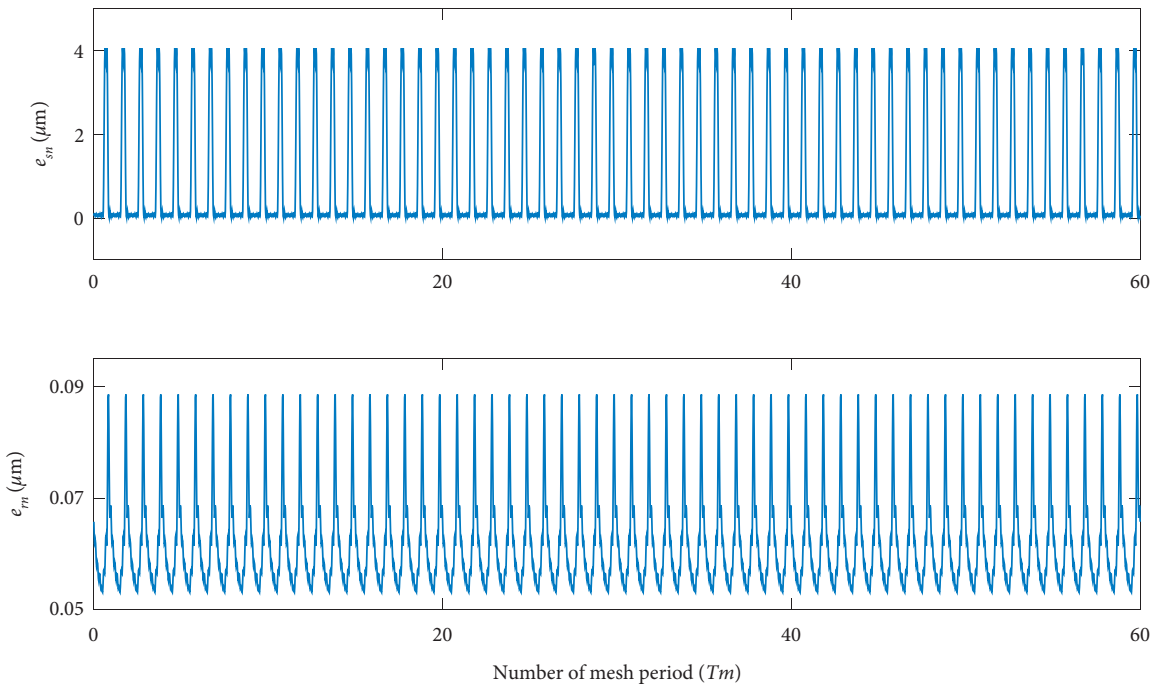

(c)

FiguRe 3: (a) External and internal mesh stiffness. (b) Enlarged drawing of the external mesh stiffness and detailed description of broken sun gear. (c) Static transmission error. 


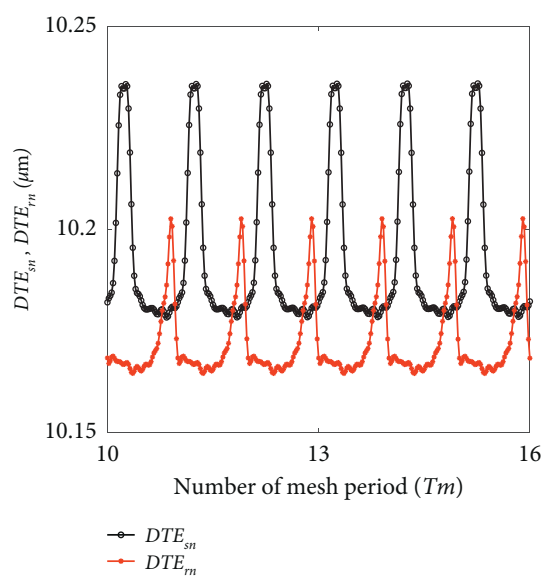

(a)

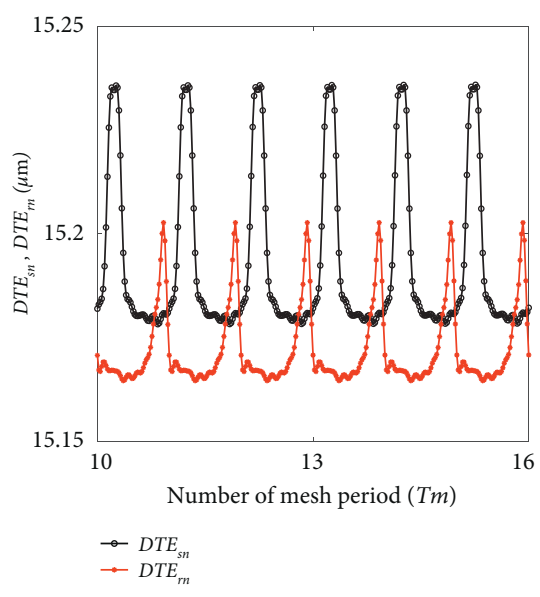

(b)

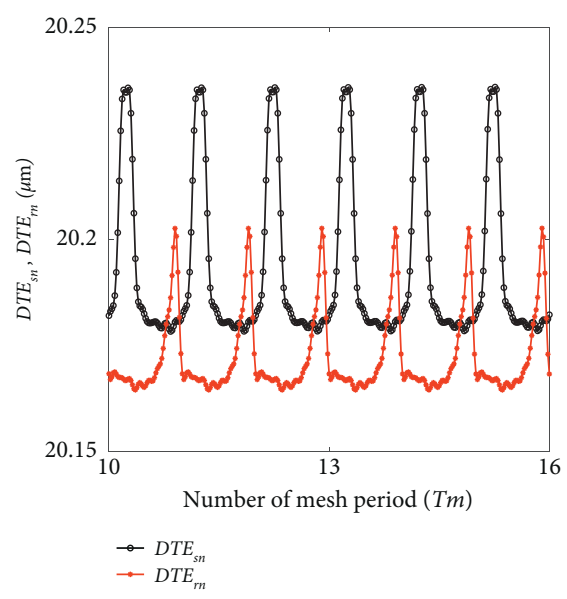

(c)

Figure 4: Dynamic transmission error. (a) $b_{s n}=b_{r n}=20 \mu \mathrm{m}$. (b) $b_{s n}=b_{r n}=30 \mu \mathrm{m}$. (c) $b_{s n}=b_{r n}=40 \mu \mathrm{m}$.

decreases. In order to better see the influence of this broken tooth in the meshing stiffness, the enlarged drawing of the external mesh stiffness is shown in Figure 3(b).

The internal mesh static transmission error $\left(e_{\mathrm{rn}}\right)$ and external mesh static transmission error $\left(e_{s n}\right)$ are consistent with those in the experiment rig in Section 4, which is shown in Figure 3(c).

\subsection{Effect Analysis of Gear Backlash and Damping. The effect} of gear backlash on the transmission of planetary gear train can be significantly reflected by dynamic transmission error under different gear backlash. The external mesh dynamic transmission error is denoted by $D T E_{s n}$, and the internal mesh dynamic transmission error is denoted by $D T E_{r n}$.

$\left\{\begin{array}{l}D T E_{s n}=\left(x_{p n}-x_{s}\right) \sin \beta_{s n}+\left(y_{s}-y_{p n}\right) \cos \beta_{s n}+u_{s}+u_{p n}, \\ D T E_{r n}=\left(x_{p n}-x_{r}\right) \sin \beta_{r n}+\left(y_{r}-y_{p n}\right) \cos \beta_{r n}+u_{r}-u_{p n} .\end{array}\right.$

Herein, variation of static transfer error is not considered and set : $b_{s n}=b_{r n}=20 \mu m, \quad b_{s n}=b_{r n}=30 \mu m$, and $b_{s n}=b_{r n}=40 \mu \mathrm{m}$. By solving the dynamics equation, $D T E_{s n}$ and $D T E_{r n}$ are obtained under these three values, and the comparative results are shown in Figure 4 . When gear backlash values are equal to $20 \mu \mathrm{m}, 30 \mu \mathrm{m}, 40 \mu \mathrm{m}$ respectively, the mean values of $D T E_{s n}$ are equal to $10.19 \mu \mathrm{m}$, $15.19 \mu \mathrm{m}$ and $20.19 \mu \mathrm{m}$ respectively. The mean of $D T E_{r n}$ is less than the mean of $D T E_{s n}$ under the same gear backlash, but it shows the same change rule as $D T E_{s n}$ for different gear backlash values. Therefore, it can be concluded that the larger gear backlash leads to the greater dynamic transmission error. The large transmission error indicates that the transmission accuracy of planetary gear train decreases. However, on the other hand, gear backlash is also indispensable for lubricating oil storage. Therefore, the actual planetary gear train should have appropriate gear backlash to store the lubricating oil, and the existence of lubricating oil can decrease the gear backlash and friction between meshing gear pair, thus reducing the dynamic transmission error and improving the transmission precision.

In this article, the damping equals $\widehat{C}=34.616 M+2.2942 \times 10^{-5}\left(K_{B}+K_{M}\right)$ for matching the values in the experiment rig in Section 4. In order to analyze the effect of damping on the system, two additional damping values, $0.0167 \widehat{C}$ and $3 \widehat{C}$, are analyzed. The dynamic equations of three kinds of damping are solved to obtain their vibration velocity on the external and internal meshing lines, denoted as $\dot{\delta}_{s n}, \dot{\delta}_{r n}$. The comparison results are shown in Figure 5.

When the damping equals to $0.0167 \widehat{C}$, the root mean square (RMS) and variance of $\dot{\delta}_{s n}$ are 5.67 times and 32.16 times those of $\widehat{C}$, respectively. Simultaneously, the RMS and variance of $\dot{\delta}_{r n}$ are 3.29 times and 10.86 times those of $\widehat{C}$, respectively. As a comparison, when the damping equals to $3 \widehat{C}$, RMS and variance of $\dot{\delta}_{s n}$ and $\dot{\delta}_{r n}$ are both 0.96 times and 0.92 times those of $\widehat{C}$, respectively.

The results show that the damping has a significant effect on the vibration and stability of the system, and small damping is detrimental to the system's vibration. When the damping equals $0.0167 \widehat{C}$, RMS and variance of $\dot{\delta}_{s n}, \dot{\delta}_{r n}$ are much greater than those of $\widehat{C}$. However, when the damping equals $3 \widehat{C}$, RMS and variance of $\dot{\delta}_{s n}, \dot{\delta}_{r n}$ are slightly less than those of $\hat{C}$. Therefore, the vibration intensity of planetary gear train with small damping is large, and its stability is poor. With the increase of damping, the vibration velocity of the system decreases, and the vibration stability of the system becomes better.

\subsection{Velocity Signal Analysis in the Healthy and Faulty State.} When the tooth breaks, the vibration velocity of each component of the planetary gear train is significantly different from that in the healthy state. Herein, vibration velocity of the sun gear $\left(\dot{y}_{s}\right)$ and the meshing velocity between the sun gear and the planetary gear $\left(\dot{\delta}_{s n}\right)$ are taken as an example, and the comparative results are shown in Figures (6)-(9).

According to Figure $6, \dot{y}_{s}$ appears as a periodic vibration with a period of $T m$ for the healthy mode ( $T m$ refers to the mesh period), which is mainly caused by the change in the 


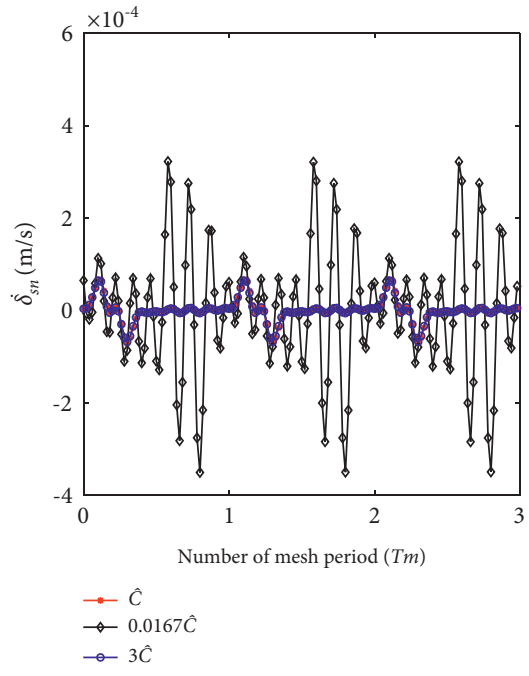

(a)

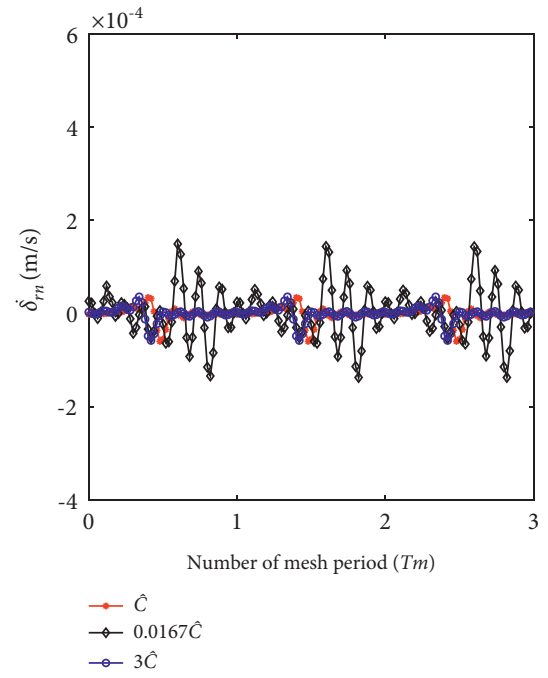

(b)

Figure 5: Vibration velocity $\left(\dot{\delta}_{s n}\right.$ and $\left.\dot{\delta}_{r n}\right)$ under different damping states.

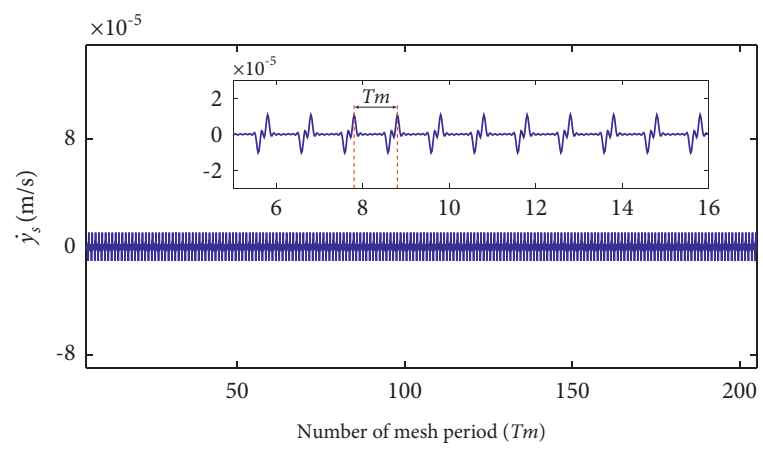

(a)

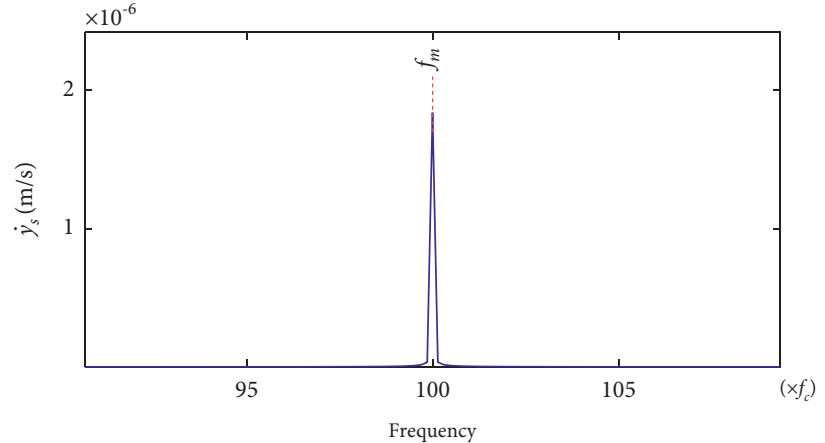

(b)

FIGURE 6: Waveform and frequency spectrum of $\dot{y}_{s}$ with a healthy sun gear.

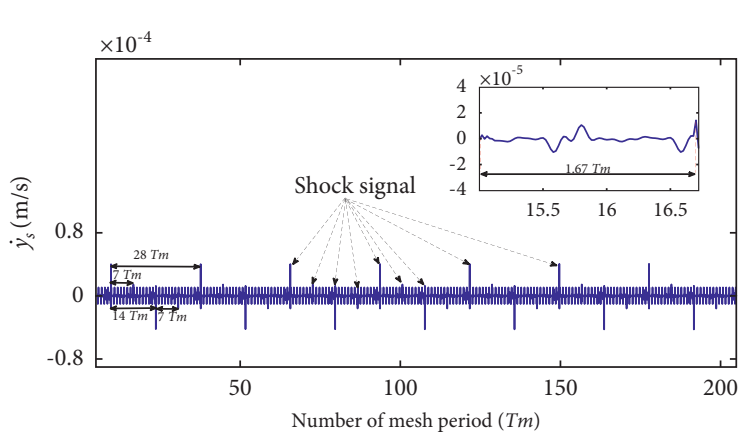

(a)

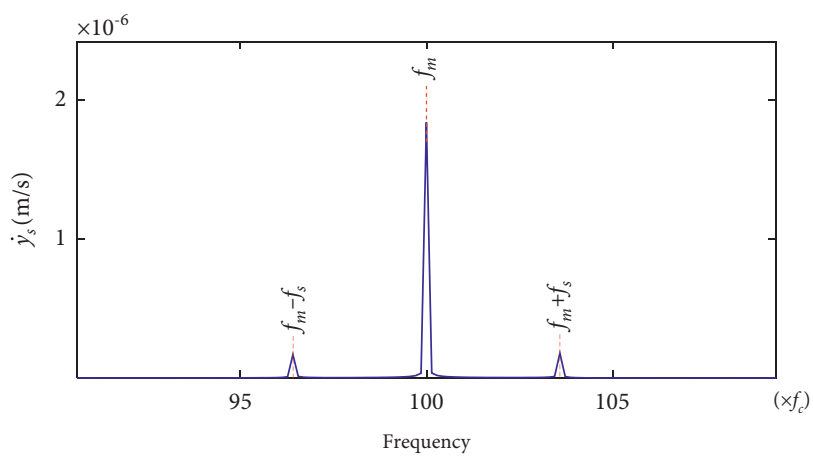

(b)

Figure 7: Waveform and frequency spectrum of $\dot{y}_{s}$ with a $2.4 \mathrm{~mm}$ broken tooth on the sun gear.

time-varying mesh stiffness. Frequency domain signals are dominated by the mesh frequency $\left(f_{m}=100 f_{c}\right)$. It is worth noting that no sideband is present around the mesh frequency.
The effect of a $2.4 \mathrm{~mm}$ broken tooth on the sun gear can be seen in Figure 7. Shock vibration with a period of $7 \mathrm{kTm}(k$ is an integer) is observed, except for the periodic vibration with a period of $\mathrm{Tm}$. The main reason is that the broken 


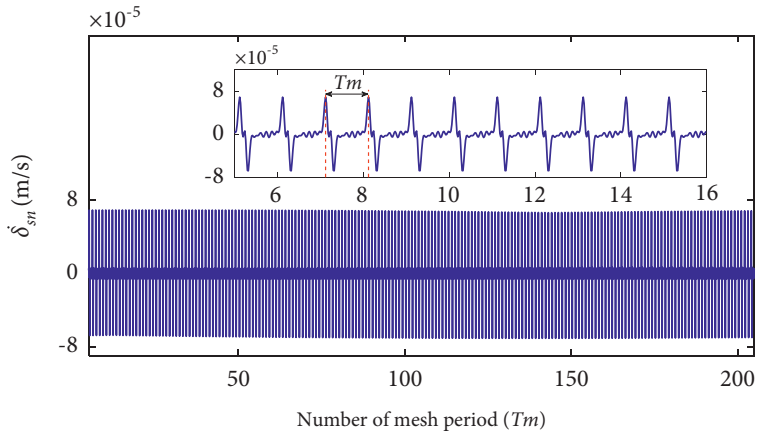

(a)

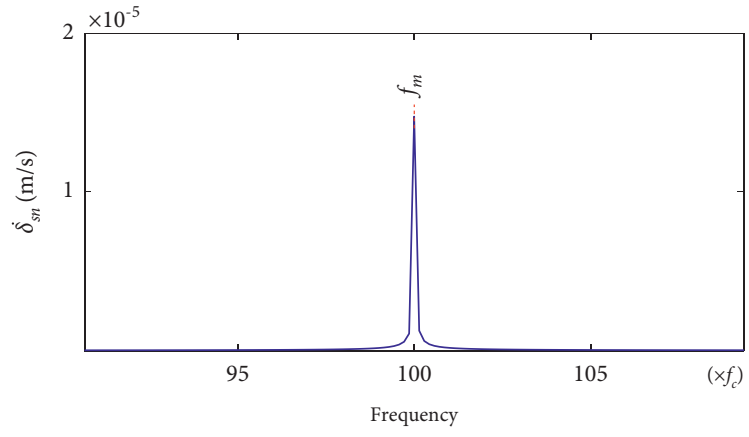

(b)

Figure 8: Waveform and frequency spectrum of $\dot{\delta}_{s n}$ with a healthy sun gear.

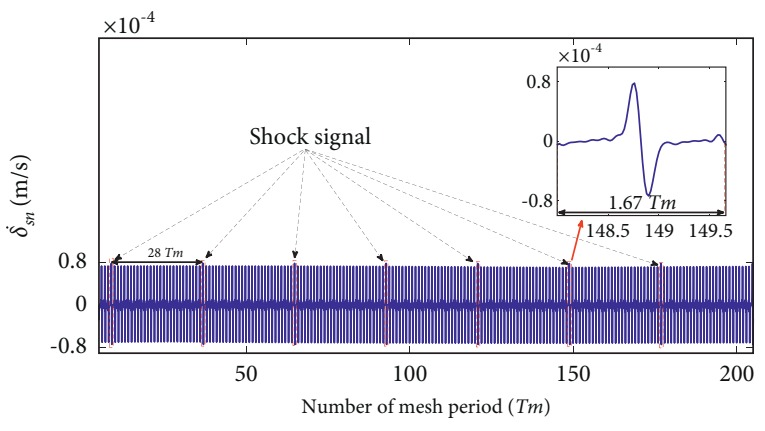

(a)

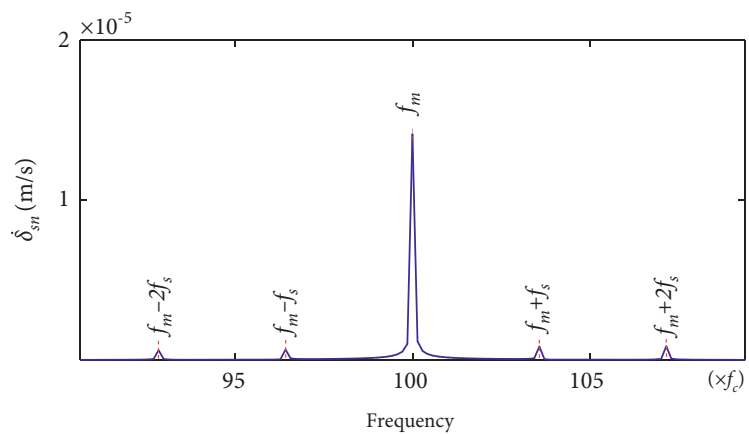

(b)

Figure 9: Waveform and frequency spectrum of $\dot{\delta}_{s n}$ with a $2.4 \mathrm{~mm}$ broken tooth on the sun gear.

tooth on the sun gear engages with four planetary gears in turn every $7 \mathrm{Tm}$ periods. Since the broken tooth has smaller mesh stiffness, an increase in $\dot{y}_{s}$ is observed. The duration time of each shock signal is $1.67 \mathrm{Tm}$, which is consistent with the contact degree of the sun-planet meshing pairs. Although frequency domain signals are always dominated by the mesh frequency, there are sideband signals around the mesh frequency with the sideband interval $f_{s}=f_{m} / z_{s}=f_{m} / 28$, where $z_{s}$ refers to the tooth number of sun gear.

According to Figure 8, the vibration velocity along the meshing line $\left(\dot{\delta}_{s n}\right)$ appears as a periodic vibration with a period of $\mathrm{Tm}$ for the healthy state, which is mainly caused by the time-varying mesh stiffness. The frequency domain signals are also dominated by the mesh frequency, and there is no sideband around the mesh frequency. By comparing Figures 6 and 8, it is observed that the intensity of $\dot{\delta}_{s n}$ is greater than that of $\dot{y}_{s}$.

The effect of a broken tooth on the sun gear can also be seen in Figure 9, and shock vibration with a period of $28 \mathrm{kTm}$ ( $k$ is an integer) is observed, except for the periodic vibration with a period of $\mathrm{Tm}$. The main reason is that the broken tooth on the sun gear engages with the same planetary gear every $28 \mathrm{Tm}$. Since the broken tooth has smaller mesh stiffness, $\dot{\delta}_{s n}$ is increased. The duration time of each shock signal equals $1.67 \mathrm{Tm}$, which is consistent with the contact degree of the sun-planet meshing pairs. Although the frequency domain signals are always dominated by the mesh frequency, there are sideband signals around mesh frequency with the sideband interval of $f_{s}$.

\section{Establishment and Analysis of the Resultant Acceleration Signal}

3.1. Acceleration Evaluation in the Fixed Coordinate System. The above analysis in Section 2 was built on the rotating coordinate system $O x y$. However, the transducer-perceived acceleration vibration signal is measured in the fixed coordinate system OXY. Herein, let the two coordinate components of the displacement vector be $x$ and $y$ in the rotating coordinate system $O x y$, and let the two coordinate components of the displacement vector be $X$ and $Y$ in the fixed coordinate system $O X Y$.

Displacement vectors satisfy the following relationship:

$$
\left\{\begin{array}{l}
x=Y \sin \omega_{c} t+X \cos \omega_{c} t \\
y=Y \cos \omega_{c} t-X \sin \omega_{c} t
\end{array}\right.
$$

Velocity vectors satisfy the following relationship:

$\left\{\begin{array}{l}\dot{x}=\dot{Y} \sin \omega_{c} t+\omega_{c} Y \cos \omega_{c} t+\dot{X} \cos \omega_{c} t-\omega_{c} X \sin \omega_{c} t \\ \dot{y}=\dot{Y} \cos \omega_{c} t-\omega_{c} Y \sin \omega_{c} t-\omega_{c} X \cos \omega_{c} t-\dot{X} \sin \omega_{c} t\end{array}\right.$ 
where $\dot{x}, \dot{X}, \dot{y}, \dot{Y}$ refer to the first derivatives of $x, X, y, Y$, respectively.
Acceleration vectors satisfy the following relationship:

$$
\left\{\begin{array}{l}
\ddot{x}=\ddot{Y} \sin \omega_{c} t+2 \omega_{c} \dot{Y} \cos \omega_{c} t-\omega_{c}^{2} Y \sin \omega_{c} t+\ddot{X} \cos \omega_{c} t-2 \omega_{c} \dot{X} \sin \omega_{c} t-\omega_{c}^{2} X \cos \omega_{c} t, \\
\ddot{y}=\ddot{Y} \cos \omega_{c} t-2 \omega_{c} \dot{Y} \sin \omega_{c} t-\omega_{c}^{2} Y \cos \omega_{c} t-\ddot{X} \sin \omega_{c} t-2 \omega_{c} \dot{X} \cos \omega_{c} t+\omega_{c}^{2} X \sin \omega_{c} t,
\end{array}\right.
$$

where $\ddot{x}, \ddot{X}, \ddot{y}, \ddot{Y}$ refer to the second derivatives of $x, X, y, Y$, respectively. Acceleration evaluation in the fixed coordinate system can be obtained by using equations (10)-(12).

\subsection{Establishment of the Resultant Acceleration Signal.} The resultant acceleration signal along the vertical direction is constructed as the weighted vibration summation. The schematic diagram of vertical vibration composition is shown in Figure 10(a). It mainly consists of five vibrational components as follows:

(1) Vertical component of the vibration along the meshing lines of sun-planet pairs. The gear mesh diagram is shown in Figure 10(b) [17]. The sun gear and the planetary gears contact and mesh with each other. They can cause vibration along the outer meshing line. Vertical component of the vibration along the meshing lines of sun-planet pairs constitutes the first term of the resultant signal.

(2) Vertical component of the vibration along the meshing lines of ring-planet pairs. As shown in Figure 10(b), the planetary gears contact the ring gear and mesh with each other. Thus, they can cause vibration along the inner meshing line. Vertical component of the vibration along the meshing lines of ring-planet pairs constitutes the second term of the resultant signal.

(3) Vertical component of the planet gears relative to their supporting bearing. The planetary gears contact with their support bearing. Thus, they can cause the corresponding vibration. Vertical component of the planet gears relative to their supporting bearing constitutes the third term of the resultant signal. This is different from that in literature $[17,21]$, where this component has not been taken into account.
(4) Vertical component of the sun gear relative to its supporting bearing. The sun gear is consolidated to its supporting shaft, which contacts with its supporting bearing. Thus, they can cause the corresponding vibration. Vertical component of the sun gear relative to its supporting bearing constitutes the fourth term of the resultant signal. This is consistent with that in [17], where sun gear's vibration was also considered as one component of the vertical vibration signals.

(5) Vertical component of the planet carrier relative to its supporting bearing. The planet carrier contacts with its support bearing. This contact can cause the corresponding vibration. Vertical component of the planet carrier relative to its supporting bearing constitutes the fifth term of the resultant signal. It is different from that in literatures [17, 21], where this component has not been taken into account.

According to [17-21], the attenuation effect of the transmission path for the vibration signal should be considered. Therefore, it is needed to build a transmission path function that reflects the path variability. A method to judge the path function's validity is to construct the resultant signals using this function and compare this resultant signal with experimental signal. Compared the existing path functions [17-21], the modified Hamming function $\left(H_{n}(t)=\exp ^{-\left(\bmod \left(\varepsilon_{n}, 2 \pi\right)-\pi\right)^{2}}\left(0.54-0.46 \cos \varepsilon_{n}\right)\right)$ was used to establish the resultant signal, and it has well matching with the experimental signal in time domain and frequency domain. The detailed data graphs are shown in Sections 3.3 and 4 .

The resultant acceleration signal along the vertical direction is denoted as $A_{Y}$, as expressed in the following equation:

$$
A_{Y}=\left(\sum_{n=1}^{N} H_{n}\left(\lambda_{1} a_{s n} \cos \left(\alpha_{s}-\varepsilon_{n}\right)+\lambda_{2} a_{r n} \cos \left(\alpha_{r}+\varepsilon_{n}\right)+\lambda_{3} a_{c n Y}\right)\right)+\lambda_{4} a_{s Y}+\lambda_{5} a_{c Y},
$$

where $\quad H_{n}(t)=\exp ^{-\left(\bmod \left(\varepsilon_{n}, 2 \pi\right)-\pi\right)^{2}}\left(0.54-0.46 \cos \varepsilon_{n}\right)$, $\varepsilon_{n}=w_{c} t+\beta_{n}, a_{s n}$ is the vibration along the meshing lines of sun-planet pairs, $a_{r n}$ is the vibration along the meshing lines of ring-planet pairs, $a_{c n Y}$ is the vertical component of the planet gears relative to their supporting bearing, $a_{s Y}$ is the vertical component of the sun gear relative to its supporting bearing, $a_{c Y}$ is the vertical component of the planet carrier relative to its supporting bearing, and $\lambda_{i}$ is the attenuation coefficient. Based on the specific structure of the experimental rig in Section 4, the following values are obtained: $\lambda_{1}=0.5, \lambda_{2}=0.8, \lambda_{3}=0.6, \lambda_{4}=0.4$, and $\lambda_{5}=0.4$. In addition, the white Gaussian noise is added to the resultant signal based on the actual background noise. 


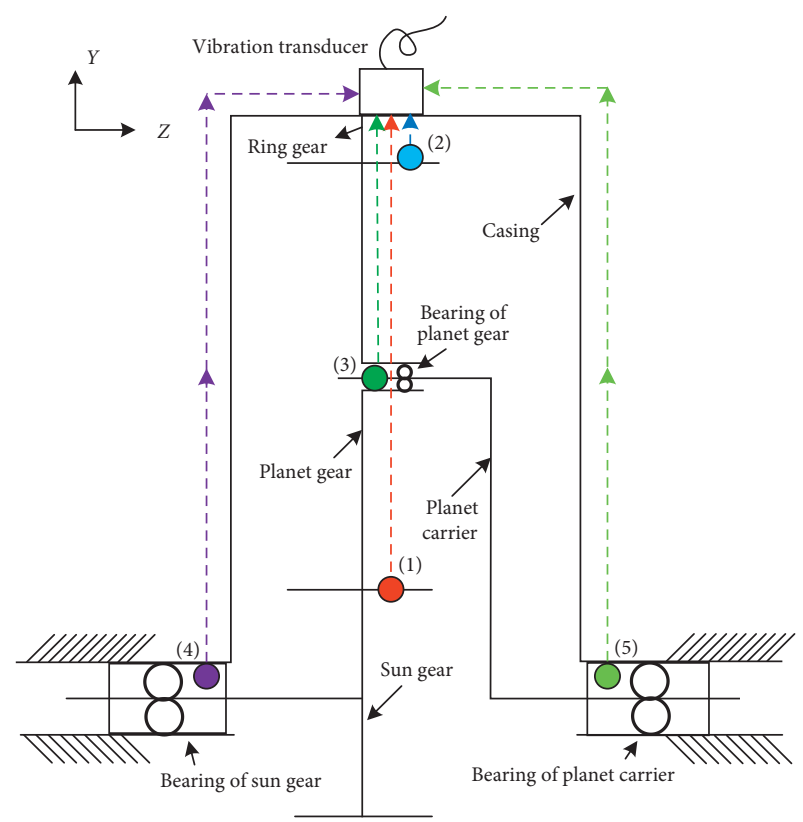

(a)

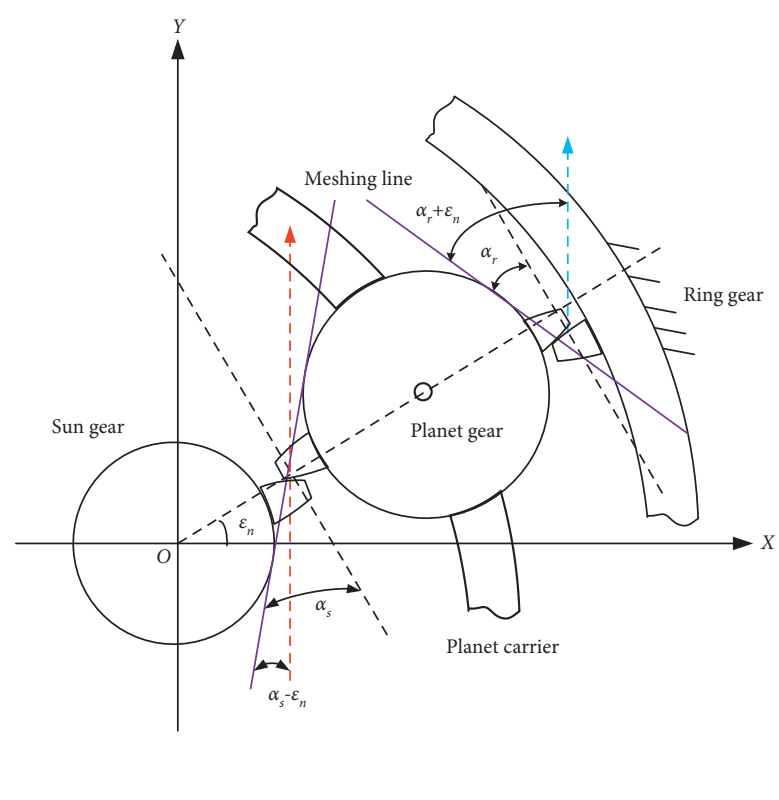

(b)

Figure 10: (a) Schematic diagram of vertical vibration composition. (b) Gear mesh diagram.

3.3. Features of the Resultant Acceleration Signal. Based on the resultant signal model developed in Section 3.2, the resultant acceleration signal is predicted and used to study the vibration features of the planetary gear train. The rotational period of the planet carrier is equal to $100 \mathrm{Tm}$. Time waveform and frequency spectra of the resultant acceleration signal with a healthy sun gear and with a $2.4 \mathrm{~mm}$ broken tooth on the sun gear are shown in Figures 11 and 12, respectively.

As shown in Figures 11(a) and 12(a), the time waveform is accompanied by the amplitude modulation in both states. Since four planets sequentially pass through the transducer location, the envelope of signals fluctuates four times in a single revolution of the planet carrier. These results are in good agreement with the results obtained in $[17,19,21]$. As shown in Figure 11(b), when the planetary gear train works with a healthy sun gear, the mesh frequency dominates the frequency spectrum with many sidebands located at $f_{m} \pm k f_{\mathrm{c}}$ ( $k$ is the integer, which has a larger amplitude when $f=f_{m} \pm l f_{c}(l=1,2,3$, and 4$\left.)\right)$. Moreover, left and right sidebands in the proximity of meshing frequency show significant asymmetric characteristics, where one of the main reasons for this asymmetric spectrum lies in the variable transmission path. Compared with the frequency spectrum of resultant vibration signals under stationary load conditions with a healthy sun gear [21], the model proposed in this article can obtain a more accurate and detailed spectrum structure.

However, after introducing a $2.4 \mathrm{~mm}$ broken tooth on the sun gear, shock signals caused by the broken tooth appear in the waveform of acceleration signals with the interval $7 \mathrm{kTm}$ ( $k$ is an integer), which is shown in Figure 12(a). As a result, some additional frequency components are included in the frequency spectrum. As shown in Figure 12(b), these sidebands are at the following locations: $f_{m} \pm k f_{s} \pm l f_{\mathrm{c}}$ (where $k$ and $l$ are integers). Furthermore, this also shows the asymmetric characteristics with more complex spectrum distributions when compared to the healthy condition. In other words, the vibration signals are also modulated by the broken tooth and the variable transmission path.

\section{Experimental Validation and Analysis}

To validate the resultant signal model above, an experimental test is performed on a planetary gear train test rig, which is shown in Figure 13(a). The test rig is composed of a programmable drive motor, a set of shaft couplings, a moment sensor, a planetary gearbox, a parallel-shaft gearbox, and a programmable brake. The planetary gearbox in the test rig is the same as the one used in the numerical simulation. The parameters of the planetary gearbox can be found in Table 1. The rotation frequency and load of the planet carrier are also consistent with those in the numerical simulation. To acquire the vibration signals, an acceleration transducer is vertically installed on the case of the planetary gearbox, while the sampling frequency is $2048 \mathrm{~Hz}$. Rolling bearings of well processing quality are installed. The healthy and faulty sun gears are the same as the ones used in the numerical simulation. They are shown in Figures 13(b) and 13(c).

Time waveform and frequency spectra of experimental vibration signal with a healthy sun gear are presented in Figure 14. As shown in Figure 14(a), there are four amplitude modulation processes in $100 \mathrm{Tm}\left(100 \mathrm{Tm}=1 / f_{c}=\mathrm{Tc}\right)$; that is to say, one rotation period of planet carrier results in four envelope fluctuations. Based on the frequency spectra of experimental vibration signals in healthy condition, the 


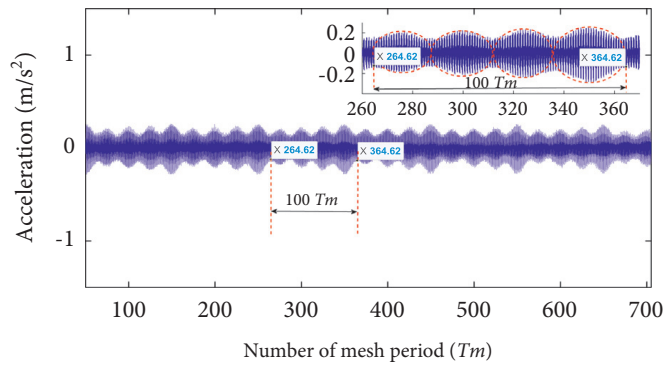

(a)

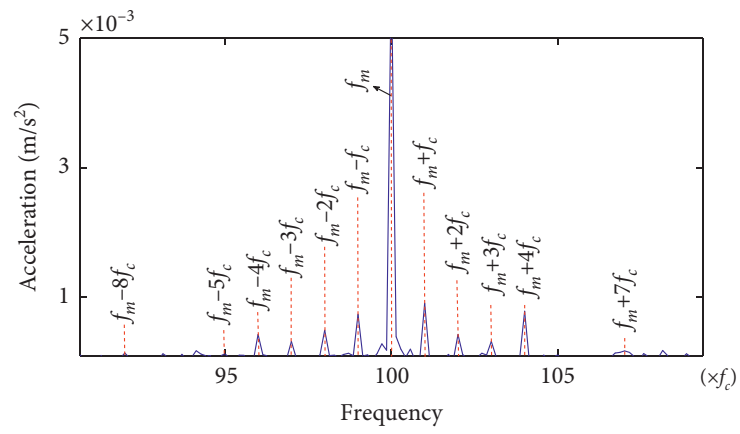

(b)

Figure 11: Time waveform and frequency spectra of $A_{Y}$ with a healthy sun gear.

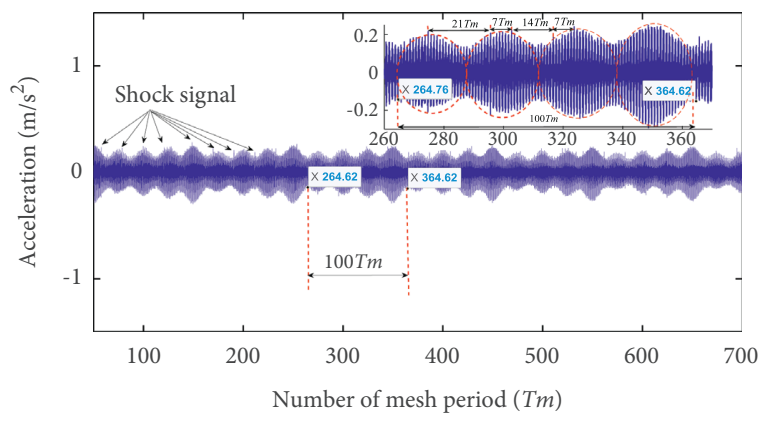

(a)

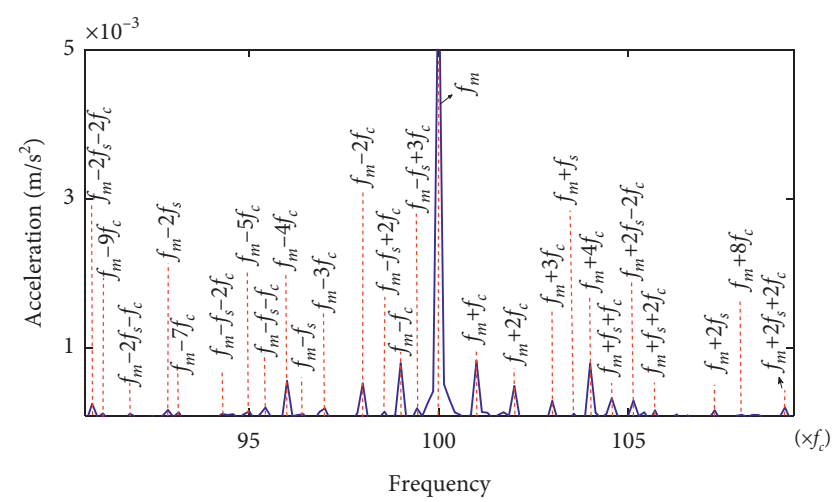

(b)

Figure 12: Time waveform and frequency spectra of $A_{Y}$ with a $2.4 \mathrm{~mm}$ broken tooth on the sun gear.

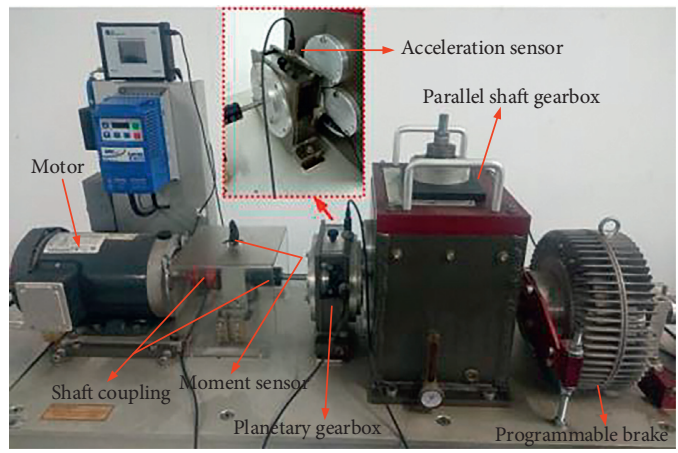

(a)

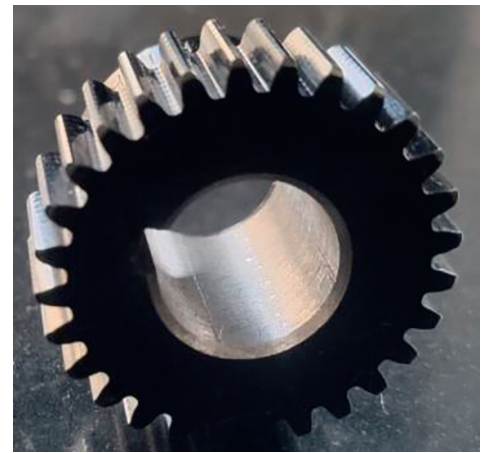

(b)

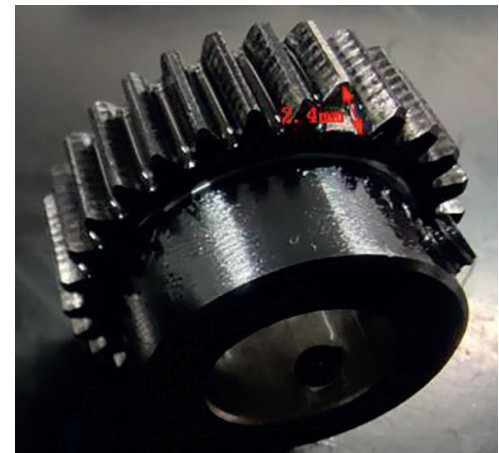

(c)

Figure 13: (a) Planetary gear train test rig. (b) Healthy sun gear. (c) A $2.4 \mathrm{~mm}$ broken tooth on the sun gear.

sidebands with large vibration amplitude locate at the $f_{m} \pm k f_{c}$ ( $k$ is an integer) centering on the meshing frequency, which is shown in Figure 14(b). Moreover, left and right sidebands around meshing frequency also show the asymmetric characteristics. The sidebands have a larger amplitude when $f=f_{m} \pm k f_{c}(k=1,2,3$, and 4). This is in agreement with the resultant acceleration signal. In addition, due to the unavoidable manufacturing errors of test rig, the vibration contains the certain shock signals even in healthy condition. These shock signals may lead to the appearance of relatively low amplitude sidebands in the frequency spectra.

Time waveform and frequency spectra of experimental vibration signal with a $2.4 \mathrm{~mm}$ broken tooth on the sun gear are presented in Figure 15. Although the amplitude 


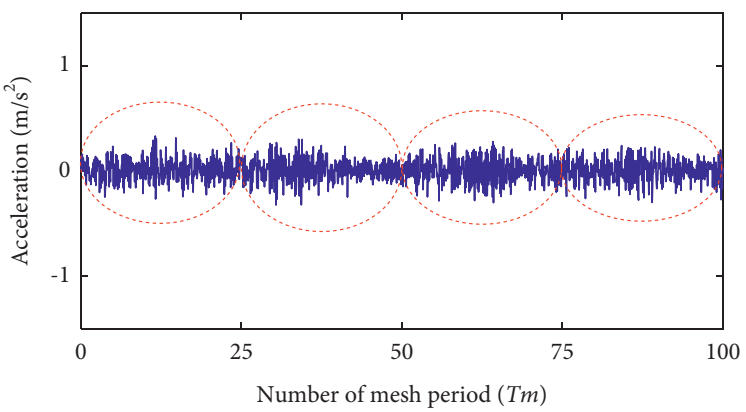

(a)

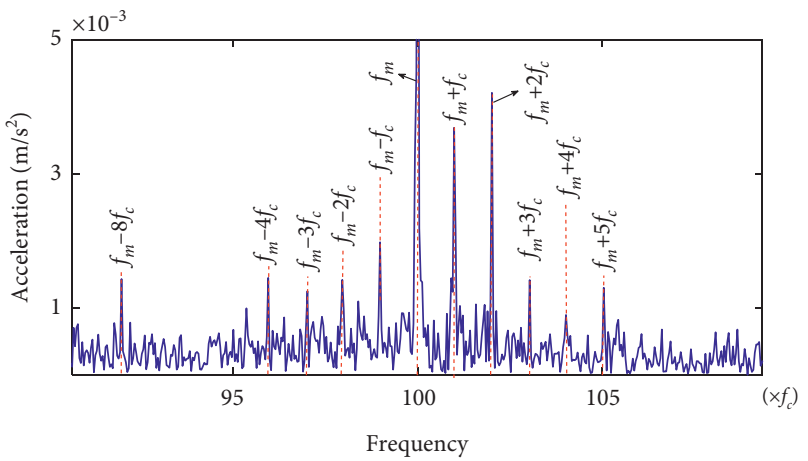

(b)

Figure 14: Time waveform and frequency spectra of experimental vibration signal with a healthy sun gear.

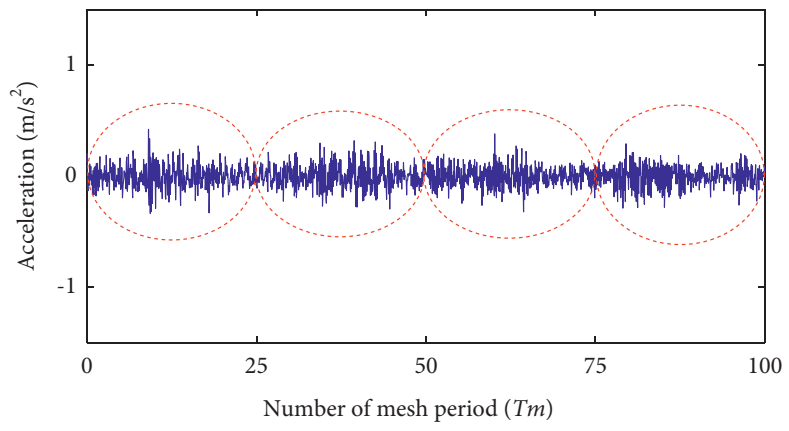

(a)

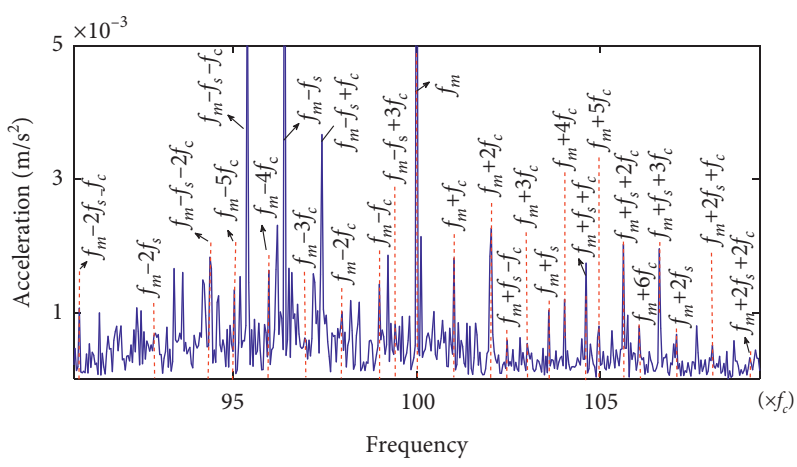

(b)

Figure 15: Time waveform and frequency spectra of experimental vibration signal with a $2.4 \mathrm{~mm}$ broken tooth on the sun gear.

modulation caused by the rotation of the planet carrier also appears in the faulty state, as shown in Figure 15(a), it can be found that the shock signal under the fault state is more obvious by comparing Figures 14(a) and 15(a). Further, the difference between the healthy and faulty state can be further found from the frequency spectra (Figures 14(b) and 15(b)). When the tooth breaks, the spectrum of the experimentally obtained acceleration signals becomes more complicated in the region of asymmetric sidebands around the mesh frequency. The sidebands with a larger amplitude appear at $f_{m} \pm k f_{s} \pm l f_{c}$ ( $k$ and $l$ are integers) centering on mesh frequency. These experimental results are consistent with the numerical simulation results obtained from the resultant vibration signal model in Section 3.

\section{Conclusions}

(1) An analytical lateral-torsional coupled dynamic model was proposed in this article with taking the multiple types nonlinear parameters into account: time-varying mesh stiffness and damping, static transmission error, and gear backlash, where computation steps of mesh stiffness using FEM are presented. Gear backlash analysis illustrates that the larger gear backlash leads to the greater dynamic transmission error. Damping analysis reveals that the damping has a significant effect on the vibration and stability of the system, and small damping is detrimental to the system's vibration. With the increase of damping, the vibration velocity of the system decreases, and the vibration stability of the system becomes better. Next, vibration velocity with and without the broken tooth on the sun gear was analyzed and compared.

(2) A new resultant acceleration signal along the vertical direction considering the effects of transmission paths was proposed. It mainly includes the vertical component of the vibration along the meshing lines of both the sun-planet and the ring-planet pairs, as well as the vertical component of the planet gears, the sun gear, and the planet carrier acceleration relative to their supporting bearings.

(3) Features of the resultant acceleration signal in healthy and faulty conditions were revealed, and an experimental rig was set up to validate the vibration characteristics simulated from the resultant vibration signal model. The experimental results indicate that the proposed resultant vibration signal model can predict the healthy and faulty features more accurately in both time and frequency domains. 
(4) The different planetary gear trains have different structural forms, modal shapes, sizes, and other attributes, so it is difficult to use a path function to adapt to all planetary gear trains. In other words, a path function that is a good match for one planetary train may not be a good match for another planetary train. In the process of research, appropriate path functions should be constructed according to the actual planetary gear train's characteristics. The significance of the path function used in this article is that it provides other researchers with a possible scheme or a way to construct the path function.

(5) The load of planetary gear train is constant in this article, and the broken tooth occurs on the sun gear. The following research will focus on the vibration features and fault identification methods of planetary gear train under variable load conditions.

\section{Appendix}

$$
\begin{aligned}
& M=\operatorname{diag}\left[M_{s}, M_{r}, M_{c}, M_{p 1}, M_{p 2}, \ldots M_{p N}\right] \text {, where, } M_{i}=\operatorname{diag}\left[m_{i}, m_{i}, I_{i} / r_{i}^{2}\right], i=s, r, c, p 1, \ldots, p N \\
& \phi=\left[x_{s}, y_{s}, u_{s}, x_{r}, y_{r}, u_{r}, x_{c}, y_{c}, u_{c}, x_{p 1}, y_{p 1}, u_{p 1}, x_{p 2}, y_{p 2}, u_{p 2} \ldots x_{p N}, y_{p N}, u_{p N}\right]^{\mathrm{T}} \text {, } \\
& W=\operatorname{diag}\left[W_{s}, W_{r}, W_{c}, W_{p 1}, W_{p 2}, \ldots, W_{p N}\right] \text {, where, } W_{i}=\left[\begin{array}{ccc}
0 & -2 m_{i} & 0 \\
2 m_{i} & 0 & 0 \\
0 & 0 & 0
\end{array}\right], i=s, r, c, p 1, \ldots, p N \text {, } \\
& K_{B}=\operatorname{diag}\left[K_{s b}, K_{r b}, K_{c b}, \mathbf{0}, \ldots, \mathbf{0}\right], \text { where }, K_{i b}=\operatorname{diag}\left[k_{i}, k_{i}, k_{i u}\right], i=s, r, c \text {, } \\
& K_{C}=\operatorname{diag}\left[K_{s c}, K_{r c}, K_{c c}, K_{p 1 c}, K_{p 2 c}, \ldots, K_{p N c}\right] \text {, where, } K_{i c}=\operatorname{diag}\left[m_{i}, m_{i}, 0\right], i=s, r, c, p 1, \ldots, p N \text {, } \\
& K_{M}=\left[\begin{array}{ccccccc}
K_{1} & \mathbf{0} & \mathbf{0} & K_{7}(1) & K_{7}(2) & \cdots & K_{7}(N) \\
\mathbf{0} & K_{3} & \mathbf{0} & K_{8}(1) & K_{8}(2) & \cdots & K_{8}(N) \\
\mathbf{0} & \mathbf{0} & K_{5} & K_{9}(1) & K_{9}(2) & \cdots & K_{9}(N) \\
K_{2}(1) & K_{4}(1) & K_{6}(1) & K_{10}(1) & \mathbf{0} & \cdots & \mathbf{0} \\
K_{2}(2) & K_{4}(2) & K_{6}(2) & \mathbf{0} & K_{10}(2) & \cdots & \mathbf{0} \\
\vdots & \vdots & \vdots & \vdots & \vdots & \ddots & \vdots \\
K_{2}(N) & K_{4}(N) & K_{6}(N) & \mathbf{0} & \mathbf{0} & \mathbf{0} & K_{10}(N)
\end{array}\right],
\end{aligned}
$$

where

$$
\begin{aligned}
K_{1}= & \sum_{n=1}^{N}\left[\begin{array}{ccc}
k_{s n}(t) \sin ^{2} \beta_{s n} & -k_{s n}(t) \sin \beta_{s n} \cos \beta_{s n} & -k_{s n}(t) \sin \beta_{s n} \\
-k_{s n}(t) \sin \beta_{s n} \cos \beta_{s n} & k_{s n}(t) \cos ^{2} \beta_{s n} & k_{s n}(t) \cos \beta_{s n} \\
-k_{s n}(t) \sin \beta_{s n} & k_{s n}(t) \cos \beta_{s n} & k_{s n}(t)
\end{array}\right] \\
K_{2}(n)= & {\left[\begin{array}{ccc}
-k_{s n}(t) \sin ^{2} \beta_{s n} & k_{s n}(t) \sin \beta_{s n} \cos \beta_{s n} & k_{s n}(t) \sin \beta_{s n} \\
k_{s n}(t) \sin \beta_{s n} \cos \beta_{s n} & -k_{s n}(t) \cos ^{2} \beta_{s n} & -k_{s n}(t) \cos \beta_{s n} \\
-k_{s n}(t) \sin \beta_{s n} & k_{s n}(t) \cos \beta_{s n} & k_{s n}(t)
\end{array}\right], n=1,2,3, \ldots, N } \\
K_{3}= & \sum_{n=1}^{N}\left[\begin{array}{ccc}
k_{r n}(t) \sin ^{2} \beta_{r n} & -k_{r n}(t) \sin \beta_{r n} \cos \beta_{r n} & -k_{r n}(t) \sin \beta_{r n} \\
-k_{r n}(t) \sin \beta_{r n} \cos \beta_{r n} & k_{r n}(t) \cos ^{2} \beta_{r n} & k_{r n}(t) \cos \beta_{r n} \\
-k_{r n}(t) \sin \beta_{r n} & k_{r n}(t) \cos \beta_{r n} & k_{r n}(t)
\end{array}\right] \\
K_{4}(n)= & {\left[\begin{array}{ccc}
-k_{r n}(t) \sin \beta_{r n} & k_{r n}(t) \sin \beta_{r n} \cos \beta_{r n} & k_{r n}(t) \sin \beta_{r n} \\
k_{r n}(t) \sin \beta_{r n} \cos \beta_{r n} & -k_{r n}(t) \cos \beta_{r n} & -k_{r n}(t) \cos \beta_{r n} \\
k_{r n}(t) \sin \beta_{r n} & -k_{r n}(t) \cos \beta_{r n} & -k_{r n}(t)
\end{array}\right], n=1,2,3, \ldots, N }
\end{aligned}
$$




$$
\begin{aligned}
& K_{5}=\sum_{n=1}^{N}\left[\begin{array}{ccc}
k_{p} & 0 & -k_{p} \sin \beta_{n} \\
0 & k_{p} & k_{p} \cos \beta_{n} \\
-k_{p} \sin \beta_{n} & k_{p} \cos \beta_{n} & k_{p}
\end{array}\right] \\
& K_{6}(n)=\left[\begin{array}{ccc}
-k_{p} & 0 & k_{p} \sin \beta_{n} \\
0 & -k_{p} & -k_{p} \cos \beta_{n} \\
0 & 0 & 0
\end{array}\right], n=1,2,3, \ldots, N \\
& K_{7}(n)=\left[\begin{array}{ccc}
-k_{s n}(t) \sin ^{2} \beta_{s n} & k_{s n}(t) \sin \beta_{s n} \cos \beta_{s n} & -k_{s n}(t) \sin \beta_{s n} \\
\left.k_{s n} t\right) \sin \beta_{s n} \cos \beta_{s n} & -k_{s n}(t) \cos ^{2} \beta_{s n} & k_{s n}(t) \cos \beta_{s n} \\
k_{s n}(t) \sin \beta_{s n} & -k_{s n}(t) \cos \beta_{s n} & k_{s n}(t)
\end{array}\right], n=1,2,3, \ldots, N \\
& K_{8}(n)=\left[\begin{array}{ccc}
-k_{r n}(t) \sin ^{2} \beta_{r n} & k_{r n}(t) \sin \beta_{r n} \cos \beta_{r n} & k_{r n}(t) \sin \beta_{r n} \\
k_{r n}(t) \sin \beta_{r n} \cos \beta_{r n} & -k_{r n}(t) \cos ^{2} \beta_{r n} & -k_{r n}(t) \cos \beta_{r n} \\
k_{r n}(t) \sin \beta_{r n} & -k_{r n}(t) \cos \beta_{r n} & -k_{r n}(t)
\end{array}\right], n=1,2,3, \ldots, N \\
& K_{9}(n)=\left[\begin{array}{ccc}
-k_{p} & 0 & 0 \\
0 & -k_{p} & 0 \\
k_{p} \sin \beta_{n} & -k_{p} \cos \beta_{n} & 0
\end{array}\right], n=1,2,3, \ldots, N \\
& K_{10}(n)=\left[\begin{array}{lll}
A_{n} & B_{n} & D_{n}
\end{array}\right], n=1,2,3, \ldots, N \\
& A_{n}=\left[\begin{array}{c}
k_{s n}(t) \sin ^{2} \beta_{s n}+k_{r n}(t) \sin ^{2} \beta_{r n}+k_{p} \\
-k_{s n}(t) \sin \beta_{s n} \cos \beta_{s n}-k_{r n}(t) \sin \beta_{r n} \cos \beta_{r n} \\
k_{s n}(t) \sin \beta_{s n}-k_{r n}(t) \sin \beta_{r n}
\end{array}\right] \\
& B_{n}=\left[\begin{array}{c}
-k_{s n}(t) \sin \beta_{s n} \cos \beta_{s n}-k_{r n}(t) \sin \beta_{r n} \cos \beta_{r n} \\
k_{s n}(t) \cos ^{2} \beta_{s n}+k_{r n}(t) \cos ^{2} \beta_{r n}+k_{p} \\
-k_{s n}(t) \cos \beta_{s n}+k_{r n}(t) \cos \beta_{r n}
\end{array}\right] \\
& D_{n}=\left[\begin{array}{c}
k_{s n}(t) \sin \beta_{s n}-k_{r n}(t) \sin \beta_{r n} \\
-k_{s n}(t) \cos \beta_{s n}+k_{r n}(t) \cos \beta_{r n} \\
k_{s n}(t)+k_{r n}(t)
\end{array}\right] \\
& C_{B}=\operatorname{diag}\left[C_{s b}, C_{r b}, C_{c b}, \mathbf{0}, \cdots, \mathbf{0}\right], \text { where, } C_{i b}=\operatorname{diag}\left[c_{i}, c_{i}, c_{i u}\right], i=s, r, c \\
& C_{M}=\left[\begin{array}{ccccccc}
C_{1} & \mathbf{0} & \mathbf{0} & C_{7}(1) & C_{7}(2) & \cdots & C_{7}(N) \\
\mathbf{0} & C_{3} & \mathbf{0} & C_{8}(1) & C_{8}(2) & \cdots & C_{8}(N) \\
\mathbf{0} & \mathbf{0} & C_{5} & C_{9}(1) & C_{9}(2) & \cdots & C_{9}(N) \\
C_{2}(1) & C_{4}(1) & C_{6}(1) & C_{10}(1) & \mathbf{0} & \cdots & \mathbf{0} \\
C_{2}(2) & C_{4}(2) & C_{6}(2) & \mathbf{0} & C_{10}(2) & \cdots & \mathbf{0} \\
\vdots & \vdots & \vdots & \vdots & \vdots & \ddots & \vdots \\
C_{2}(N) & C_{4}(N) & C_{6}(N) & \mathbf{0} & \mathbf{0} & \mathbf{0} & C_{10}(N)
\end{array}\right]
\end{aligned}
$$


where

$$
\begin{aligned}
& C_{1}=\sum_{n=1}^{N}\left[\begin{array}{ccc}
c_{s n}(t) \sin ^{2} \beta_{s n} & -c_{s n}(t) \sin \beta_{s n} \cos \beta_{s n} & -c_{s n}(t) \sin \beta_{s n} \\
-c_{s n}(t) \sin \beta_{s n} \cos \beta_{s n} & c_{s n}(t) \cos ^{2} \beta_{s n} & c_{s n}(t) \cos \beta_{s n} \\
-c_{s n}(t) \sin \beta_{s n} & c_{s n}(t) \cos \beta_{s n} & c_{s n}(t)
\end{array}\right] \\
& C_{2}(n)=\left[\begin{array}{ccc}
-c_{s n}(t) \sin ^{2} \beta_{s n} & c_{s n}(t) \sin \beta_{s n} \cos \beta_{s n} & c_{s n}(t) \sin \beta_{s n} \\
c_{s n}(t) \sin \beta_{s n} \cos \beta_{s n} & -c_{s n}(t) \cos ^{2} \beta_{s n} & -c_{s n}(t) \cos \beta_{s n} \\
-c_{s n}(t) \sin \beta_{s n} & c_{s n}(t) \cos \beta_{s n} & c_{s n}(t)
\end{array}\right], n=1,2,3, \ldots N \\
& C_{3}=\sum_{n=1}^{N}\left[\begin{array}{ccc}
c_{r n}(t) \sin ^{2} \beta_{r n} & -c_{r n}(t) \sin \beta_{r n} \cos \beta_{r n} & -c_{r n}(t) \sin \beta_{r n} \\
-c_{r n}(t) \sin \beta_{r n} \cos \beta_{r n} & c_{r n}(t) \cos ^{2} \beta_{r n} & c_{r n}(t) \cos \beta_{r n} \\
-c_{r n}(t) \sin \beta_{r n} & c_{r n}(t) \cos \beta_{r n} & c_{r n}(t)
\end{array}\right] \\
& C_{4}(n)=\left[\begin{array}{ccc}
-c_{r n}(t) \sin ^{2} \beta_{r n} & c_{r n}(t) \sin \beta_{r n} \cos \beta_{r n} & c_{r n}(t) \sin \beta_{r n} \\
c_{r n}(t) \sin \beta_{r n} \cos \beta_{r n} & -c_{r n}(t) \cos ^{2} \beta_{r n} & -c_{r n}(t) \cos \beta_{r n} \\
c_{r n}(t) \sin \beta_{r n} & -c_{r n}(t) \cos \beta_{r n} & -c_{r n}(t)
\end{array}\right], n=1,2,3, \ldots, N \\
& C_{5}=\sum_{n=1}^{N}\left[\begin{array}{ccc}
c_{p} & 0 & -c_{p} \sin \beta_{n} \\
0 & c_{p} & c_{p} \cos \beta_{n} \\
-c_{p} \sin \beta_{n} & c_{p} \cos \beta_{n} & c_{p}
\end{array}\right] \\
& C_{6}(n)=\left[\begin{array}{ccc}
-c_{p} & 0 & c_{p} \sin \beta_{n} \\
0 & -c_{p} & -c_{p} \cos \beta_{n} \\
0 & 0 & 0
\end{array}\right], n=1,2,3, \ldots, N \\
& C_{7}(n)=\left[\begin{array}{ccc}
-c_{s n}(t) \sin ^{2} \beta_{s n} & c_{s n}(t) \sin \beta_{s n} \cos \beta_{s n} & -c_{s n}(t) \sin \beta_{s n} \\
c_{s n}(t) \sin \beta_{s n} \cos \beta_{s n} & -c_{s n}(t) \cos ^{2} \beta_{s n} & c_{s n}(t) \cos \beta_{s n} \\
c_{s n}(t) \sin \beta_{s n} & -c_{s n}(t) \cos \beta_{s n} & c_{s n}(t)
\end{array}\right], n=1,2,3, \ldots, N \\
& C_{8}(n)=\left[\begin{array}{ccc}
-c_{r n}(t) \sin ^{2} \beta_{r n} & c_{r n}(t) \sin \beta_{r n} \cos \beta_{r n} & c_{r n}(t) \sin \beta_{r n} \\
c_{r n}(t) \sin \beta_{r n} \cos \beta_{r n} & -c_{r n}(t) \cos ^{2} \beta_{r n} & -c_{r n}(t) \cos \beta_{r n} \\
c_{r n}(t) \sin \beta_{r n} & -c_{r n}(t) \cos \beta_{r n} & -c_{r n}(t)
\end{array}\right], n=1,2,3, \ldots N \\
& C_{9}(n)=\left[\begin{array}{ccc}
-c_{p} & 0 & 0 \\
0 & -c_{p} & 0 \\
c_{p} \sin \beta_{n} & -c_{p} \cos \beta_{n} & 0
\end{array}\right], n=1,2,3, \ldots, N \\
& C_{10}(n)=\left[\begin{array}{lll}
H_{n} & I_{n} & J_{n}
\end{array}\right], n=1,2,3, \ldots, N
\end{aligned}
$$




$$
\begin{aligned}
& H_{n}=\left[\begin{array}{c}
c_{s n}(t) \sin ^{2} \beta_{s n}+c_{r n}(t) \sin ^{2} \beta_{r n}+c_{p} \\
-c_{s n}(t) \sin \beta_{s n} \cos \beta_{s n}-c_{r n}(t) \sin \beta_{r n} \cos \beta_{r n} \\
c_{s n}(t) \sin \beta_{s n}-c_{r n}(t) \sin \beta_{r n}
\end{array}\right] \\
& I_{n}=\left[\begin{array}{c}
-c_{s n}(t) \sin \beta_{s n} \cos \beta_{s n}-c_{r n}(t) \sin \beta_{r n} \cos \beta_{r n} \\
c_{s n}(t) \cos ^{2} \beta_{s n}+c_{r n}(t) \cos ^{2} \beta_{r n}+c_{p} \\
-c_{s n}(t) \cos \beta_{s n}+c_{r n}(t) \cos \beta_{r n}
\end{array}\right] \\
& J_{n}=\left[\begin{array}{c}
c_{s n}(t) \sin \beta_{s n}-c_{r n}(t) \sin \beta_{r n} \\
-c_{s n}(t) \cos \beta_{s n}+c_{r n}(t) \cos \beta_{r n} \\
c_{s n}(t)+c_{r n}(t)
\end{array}\right] \\
& F_{E}=\left[0,0, T_{s}(t) / r_{s}, 0,0,0,0,0,-T_{c}(t) / r_{c}, 0,0,0, \ldots, 0,0,0\right]^{\mathrm{T}} \\
& F_{I}=\left[\sum_{n=1}^{N} F_{s p n}, \sum_{n=1}^{N} F_{r p n}, 0,0,0, \widehat{F}_{s p 1}+\widehat{F}_{r p 1}, \ldots, \widehat{F}_{s p N}+\widehat{F}_{r p N}\right]^{\mathrm{T}}
\end{aligned}
$$

where

$$
\begin{aligned}
& F_{s p n}=\left(k_{s n}(t) e_{s n}(t)+c_{s n}(t) \dot{e}_{s n}(t)\right)\left[-\sin \beta_{s n}, \cos \beta_{s n}, 1\right], \quad n=1,2,3, \ldots, N \\
& F_{r p n}=\left(k_{r n}(t) e_{r n}(t)+c_{r n}(t) \dot{e}_{r n}(t)\right)\left[-\sin \beta_{r n}, \cos \beta_{r n}, 1\right], \quad n=1,2,3, \ldots, N \\
& \widehat{F}_{s p n}=\left(k_{s n}(t) e_{s n}(t)+c_{s n}(t) \dot{e}_{s n}(t)\right)\left[\sin \beta_{s n},-\cos \beta_{s n}, 1\right], \quad n=1,2,3, \ldots, N \\
& \widehat{F}_{r p n}=\left(k_{r n}(t) e_{r n}(t)+c_{r n}(t) \dot{e}_{r n}(t)\right)\left[\sin \beta_{r n},-\cos \beta_{r n},-1\right], \quad n=1,2,3, \ldots, N
\end{aligned}
$$

where $\dot{e}_{s n}$ and $\dot{e}_{r n}$ refer to the first derivatives of $e_{s n}$ and $e_{r n}$, respectively.

\section{Data Availability}

The data used to support the findings of this study are available from the corresponding author upon request.

\section{Conflicts of Interest}

The authors declare that there are no conflicts of interest regarding the publication of this article.

\section{Acknowledgments}

This work was supported by the National Natural Science Foundation of China (Grant no. 51605005); University Natural Science Research Key Project of Anhui Province (Grant no. KJ2020A0244); and the First Group of 2011 Plan of China's Jiangsu Province (Grant No. [2013] 56).

\section{References}

[1] A. Bodas and A. Kahraman, "Influence of carrier and gear manufacturing errors on the static load sharing behavior of planetary gear sets," JSME International Journal Series C, vol. 47, no. 3, pp. 908-915, 2004.

[2] A. Singh, "Load sharing behavior in epicyclic gears: physical explanation and generalized formulation," Mechanism and Machine Theory, vol. 45, no. 3, pp. 511-530, 2010.
[3] A. Singh, "Epicyclic load sharing map - development and validation," Mechanism and Machine Theory, vol. 46, no. 5, pp. 632-646, 2011.

[4] J. Sanchez-Espiga, A. Fernandez-Del-Rincon, M. Iglesias, and F. Viadero, "Influence of errors in planetary transmissions load sharing under different mesh phasing," Mechanism and Machine Theory, vol. 153, pp. 1-17, 2020.

[5] M. Karray, F. Chaari, A. Rincon, F. Viadero, and M. Haddar, Modulation Sidebands of Planetary Gear Set, Springer, Berlin, Germany, 2014.

[6] Z. Feng and M. J. Zuo, "Vibration signal models for fault diagnosis of planetary gearboxes," Journal of Sound and Vibration, vol. 331, no. 22, pp. 4919-4939, 2012.

[7] T. R. Pattabiraman, K. Srinivasan, and K. Malarmohan, "Assessment of sideband energy ratio technique in detection of wind turbine gear defects," Case Study in Mechanical Systems and Signal Processing, vol. 2, pp. 1-11, 2015.

[8] X. Liu, "Vibration modelling and fault evolution symptom analysis of a planetary gear train for sun gear wear status assessment," Mechanical Systems and Signal Processing, vol. 166, no. 7, pp. 1-26, 2022.

[9] H. Han, Z. Zhao, H. Tian, H. Ma, and X. Li, "Fault feature analysis of planetary gear set influenced by cracked gear tooth and pass effect of the planet gears," Engineering Failure Analysis, vol. 121, pp. 1-17, 2021.

[10] G. Li, F. Li, Y. Wang, and D. Dong, "Fault diagnosis for a multistage planetary gear set using model-based simulation and experimental investigation," Shock and Vibration, vol. 2016, pp. 1-19, Article ID 9263298, 2016.

[11] L. Zhang, Y. Wang, K. Wu, R. Sheng, and Q. Huang, "Dynamic modeling and vibration characteristics of a two-stage 
closed-form planetary gear train," Mechanism and Machine Theory, vol. 97, pp. 12-28, 2016.

[12] W. Bartelmus, F. Chaari, R. Zimroz, and M. Haddar, "Modelling of gearbox dynamics under time-varying nonstationary load for distributed fault detection and diagnosis," European Journal of Mechanics - A: Solids, vol. 29, no. 4, pp. 637-646, 2010.

[13] X. Liang, M. J. Zuo, and L. Liu, "A windowing and mapping strategy for gear tooth fault detection of a planetary gearbox," Mechanical Systems and Signal Processing, vol. 80, pp. 445459, 2016.

[14] C. G. Cooley and R. G. Parker, "The geometry and frequency content of planetary gear single-mode vibration," Mechanical Systems and Signal Processing, vol. 40, no. 1, pp. 91-104, 2013.

[15] S. Xue and I. Howard, "Torsional vibration signal analysis as a diagnostic tool for planetary gear fault detection," Mechanical Systems and Signal Processing, vol. 100, pp. 706-728, 2018.

[16] Z. Cheng, N. Hu, and J. Gao, "An approach to detect damage quantitatively for planetary gear sets based on physical models," Advanced Science Letters, vol. 4, no. 4, pp. 1695-1701, 2011.

[17] Y. Lei, X. Luo, Z. Liu, F. Lu, and W. Tang, "A new dynamic model of planetary gear sets and research on fault response characteristics," Journal of Mechanical Engineering, vol. 52, no. 13, pp. 111-122, 2016.

[18] X. Liang, M. J. Zuo, and M. R. Hoseini, "Vibration signal modeling of a planetary gear set for tooth crack detection," Engineering Failure Analysis, vol. 48, pp. 185-200, 2015.

[19] L. Liu, X. Liang, and M. J. Zuo, "Vibration signal modeling of a planetary gear set with transmission path effect analysis," Measurement, vol. 85, pp. 20-31, 2016.

[20] M. Inalpolat and A. Kahraman, "A dynamic model to predict modulation sidebands of a planetary gear set having manufacturing errors," Journal of Sound and Vibration, vol. 329, no. 4, pp. 371-393, 2010.

[21] X. Liu, Y. Yang, and J. Zhang, "Resultant vibration signal model based fault diagnosis of a single stage planetary gear train with an incipient tooth crack on the sun gear," Renewable Energy, vol. 122, pp. 65-79, 2018.

[22] P. Velex and M. Maatar, "A mathematical model for analyzing the influence of shape deviations and mounting errors on gear dynamic behaviour," Journal of Sound and Vibration, vol. 191, no. 5, pp. 629-660, 1996.

[23] A. F. d. Rincon, F. Viadero, M. Iglesias, A. De-Juan, P. Garcia, and R. Sancibrian, "Effect of cracks and pitting defects on gear meshing," Proceedings of the Institution of Mechanical Engineers - Part C: Journal of Mechanical Engineering Science, vol. 226, no. 11, pp. 2805-2815, 2012.

[24] X. Liang, M. J. Zuo, and M. Pandey, “Analytically evaluating the influence of crack on the mesh stiffness of a planetary gear set," Mechanism and Machine Theory, vol. 76, no. 1, pp. 20-38, 2016.

[25] H. Ma, R. Song, X. Pang, and B. Wen, “Time-varying mesh stiffness calculation of cracked spur gears," Engineering Failure Analysis, vol. 44, pp. 179-194, 2014.

[26] K. Li, Z. Feng, and X. Liang, "Planetary gearbox fault diagnosis via torsional vibration signal analysis in resonance region," Shock and Vibration, vol. 2017, pp. 1-18, Article ID 6565237, 2017.

[27] M. Hui, Z. Li, M. Feng, R. Feng, and B. Wen, "Time-varying mesh stiffness calculation of spur gears with spalling defect," Engineering Failure Analysis, vol. 66, pp. 166-176, 2016.

[28] A. Saxena, A. Parey, and M. Chouksey, "Time varying mesh stiffness calculation of spur gear pair considering sliding friction and spalling defects," Engineering Failure Analysis, vol. 70, pp. 200-211, 2016.

[29] H. Jiang, Y. Shao, and C. K. Mechefske, "Dynamic characteristics of helical gears under sliding friction with spalling defect," Engineering Failure Analysis, vol. 39, pp. 92-107, 2014.

[30] S. A. Abouel-seoud, E. S. Dyab, and M. S. Elmorsy, "Influence of tooth pitting and cracking on gear meshing stiffness and dynamic response of wind turbine gearbox," Int. J. Sci. Adv. Technol, vol. 2, no. 3, pp. 151-165, 2012.

[31] A. Kahraman and H. Ding, "A methodology to predict surface wear of planetary gears under dynamic conditions," $\mathrm{Me}$ chanics Based Design of Structures and Machines, vol. 38, no. 4, pp. 493-515, 2010.

[32] J. Han, Y. Liu, L. Liang, Y. Zhao, and H. Zhang, "Dynamic analysis of a fault planetary gear system under nonlinear parameter excitation," Shock and Vibration, vol. 2021, pp. 117, Article ID 1787525, 2021.

[33] G. Li, X. Liang, and F. Li, "Model-based analysis and fault diagnosis of a compound planetary gear set with damaged sun gear," Journal of Mechanical Science and Technology, vol. 32, no. 7, pp. 3081-3096, 2018.

[34] Y. Lei, J. Lin, M. J. Zuo, and Z. He, "Condition monitoring and fault diagnosis of planetary gearboxes: a review," Measurement, vol. 48, pp. 292-305, 2014.

[35] H. Ma, J. Zeng, R. Feng, X. Pang, Q. Wang, and B. Wen, "Review on dynamics of cracked gear systems," Engineering Failure Analysis, vol. 55, pp. 224-245, 2015.

[36] S. Xue, R. Entwistle, I. Mazhar, and I. Howard, "The spur planetary gear torsional stiffness and its crack sensitivity under quasi-static conditions," Engineering Failure Analysis, vol. 63, pp. 106-120, 2016.

[37] X. Liang, H. Zhang, L. Liu, and M. J. Zuo, "The influence of tooth pitting on the mesh stiffness of a pair of external spur gears," Mechanism and Machine Theory, vol. 106, pp. 1-15, 2016.

[38] F. Chaari, M. S. Abbes, F. V. Rueda, A. F. del Rincon, and M. Haddar, "Analysis of planetary gear transmission in nonstationary operations," Frontiers of Mechanical Engineering, vol. 8, no. 1, pp. 88-94, 2013.

[39] M. Sang, K. Huang, Y. Xiong, G. Han, and Z. Cheng, "Dynamic modeling and vibration analysis of a cracked $3 \mathrm{~K}-\mathrm{II}$ planetary gear set for fault detection," Mechanical Sciences, vol. 12, no. 2, pp. 847-861, 2021.

[40] J. Park, J. M. Ha, H. Oh, B. D. Youn, J.-H. Choi, and N. H. Kim, "Model-based fault diagnosis of a planetary gear: a novel approach using transmission error," IEEE Transactions on Reliability, vol. 65, no. 4, pp. 1830-1841, 2016.

[41] Z. Cheng, N. Q. Hu, F. S. Gu, and G. J. Qin, "Pitting damage levels estimation for planetary gear sets based on model simulation and grey relational analysis," Transactions of the Canadian Society for Mechanical Engineering, vol. 35, no. 3, pp. 403-417, 2011.

[42] X. Liang, M. J. Zuo, and Z. Feng, "Dynamic modeling of gearbox faults: a review," Mechanical Systems and Signal Processing, vol. 98, pp. 852-876, 2018.

[43] J. Park, J. M. Ha, H. Oh, B. D. Youn, S. Park, and J. H. Choi, "Experimental approach for estimating mesh stiffness in faulty states of rotating gear," Annual Conference of the PHM Society, vol. 7, no. 1, pp. 618-624, 2015.

[44] G. He, K. Ding, W. Li, and Y. Li, "Frequency response model and mechanism for wind turbine planetary gear train vibration analysis," IET Renewable Power Generation, vol. 11, no. 4, pp. 425-432, 2017. 
[45] G. He, K. Ding, X. Wu, and X. Yang, "Dynamics modeling and vibration modulation signal analysis of wind turbine planetary gearbox with a floating sun gear," Renewable Energy, vol. 139, pp. 718-729, 2019.

[46] Y. Li, K. Ding, G. He, and X. Yang, "Vibration modulation sidebands mechanisms of equally-spaced planetary gear train with a floating sun gear," Mechanical Systems and Signal Processing, vol. 129, pp. 70-90, 2019.

[47] O. Graja, B. Zghal, K. Dziedziech et al., "Simulating the dynamic behavior of planetary gearbox based on improved Hanning function," Comptes Rendus Mecanique, vol. 347, pp. 49-61, 2019.

[48] B. Zghal, O. Graja, K. Dziedziech et al., "A new modeling of planetary gear set to predict modulation phenomenon," Mechanical Systems and Signal Processing, vol. 127, pp. 234$261,2019$.

[49] A. Kahraman and R. Singh, "Interactions between timevarying mesh stiffness and clearance non-linearities in a geared system," Journal of Sound and Vibration, vol. 146, no. 1, pp. 135-156, 1991. 